Sādhanā Vol. 37, Part 1, February 2012, pp. 33-57. (C) Indian Academy of Sciences

\title{
Modified Adomian decomposition method for fracture of laminated uni-directional composites
}

\author{
B K RAGHU PRASAD* and P V RAMANA \\ Department of Civil Engineering, Indian Institute of Science, \\ Bangalore 560 012, India \\ e-mail: bkr@civil.iisc.ernet.in
}

\begin{abstract}
In this paper, the well-known Adomian Decomposition Method (ADM) is modified to solve the fracture laminated multi-directional problems. The results are compared with the existing analytical/exact or experimental method. The already known existing ADM is modified to improve the accuracy and convergence. Thus, the modified method is named as Modified Adomian Decomposition Method (MADM). The results from MADM are found to converge very quickly, simple to apply for fracture(singularity) problems and are more accurate compared to experimental and analytical methods. MADM is quite efficient and is practically well-suited for use in these problems. Several examples are given to check the reliability of the present method. In the present paper, the principle of the decomposition method is described, and its advantages form the analyses of fracture of laminated uni-directional composites.
\end{abstract}

Keywords. Fracture of laminated uni-directional composites; initial value problems; modified Adomian decomposition method; experimental and analytical solutions.

\section{Literature review on laminated fracture}

It is surprising that the Adomian Decomposition Method (ADM) has not been applied for ordinary differential equations appearing in the fracture problem. Particularly in laminated composites, (we solved) several coupled ordinary differential equations and it appears from literature that ADM has not been applied at all. In further review, focus has been placed on the analytical and computational techniques to analyse end notched flexure (ENF) and end notched cantilever (ENC) specimens for inter-laminar fracture toughness of composites. Carlsson \& Gillespie (1989) reviewed the fracture mechanics and experimental mechanics approaches used to characterize mode II inter-laminar fracture of composites. The review covers the detailed technical aspects of analytical, numerical and experimental methods to analyse ENF specimen for the mode II inter-laminar fracture toughness.

*For correspondence 


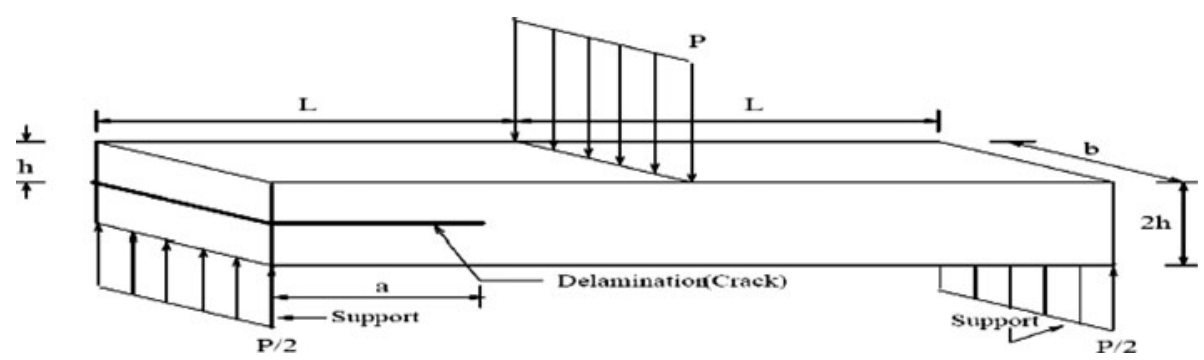

Figure 1. A schematic ENF test specimen.

\subsection{Mode II: ENF \& ENC specimens}

The short beam shear (SBS) test method was perhaps the first test method used to investigate inter-laminar fracture of composite laminates on a routine basis (Whitney et al 1974; Whitney \& Browning 1998; Whitney 1991). Introduced in the 1960s, the method still remains an important quality control test. This test method, however, has some limitations. The small span to depth ratio, in conjunction with perturbations in the stress distribution due to load introduction that do not decay rapidly in orthotropic materials, violates the beam theory based data-reduction scheme and promotes alternate failure models by Whitney \& Browning (1998), Whitney (1991). Consequently, the SBS test method measures the apparent inter-laminar shear strength of the composite. Classical linear elastic fracture mechanics (LEFM) has been used more recently to characterize the inter-laminar fracture toughness of composite materials. In contrast to the SBS test method, well-defined delaminations are embedded or machined into the specimen. Barrett \& Foschi (1977) utilized ENF specimen to characterize the mode II inter-laminar fracture of cracked wood beams. Russell \& Street (1982) used this specimen to characterize mode II critical strain energy release rates of advanced composites. The geometry of the ENF specimen is essentially a three-point flexure specimen with an embedded through-width delamination placed at the laminate mid-surface. The delamination is placed at the end of the specimen to accommodate the sliding deformation of the sub-laminates that result from the flexural loading. A typical ENF specimen is $25 \mathrm{~mm}$ wide (w), $100 \mathrm{~mm}$ long (2 L) and 3-4 mm in thickness ( $2 \mathrm{~h}$ ). Carlsson et al (1986b) presented an analysis of ENF specimen for the characterization of mode II interlaminar fracture toughness. Shear deformation plate theory mentioned in Whitney \& Pagano (1970), which incorporates a shear stress singularity at the crack tip is employed and correlated with simple beam theory expressions and finite element results for compliance and strain energy release rate of the specimen. The shear deformation plate theory solution indicates that the strain energy release rate is highly sensitive to the characteristic distance associated with the decay of the shear stress singularity to beam theory behaviour in the ENF geometry. Parametric study has been carried out to investigate the influence of geometry and material properties on compliance and strain energy release rate (figure 1 and figure 4).

\section{Stress analysis models and concepts}

The analyses of unidirectional end notched flexure and end notched cantilever specimens, using first order shear deformation beam theory(FOBT) and second order shear deformation beam theory(SOBT) theories, have been presented for mode II (shearing mode) interlaminar fracture toughness of composites, following the method adopted in the paper by 


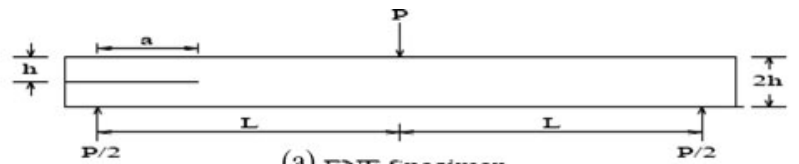

(a) ENF Specimen
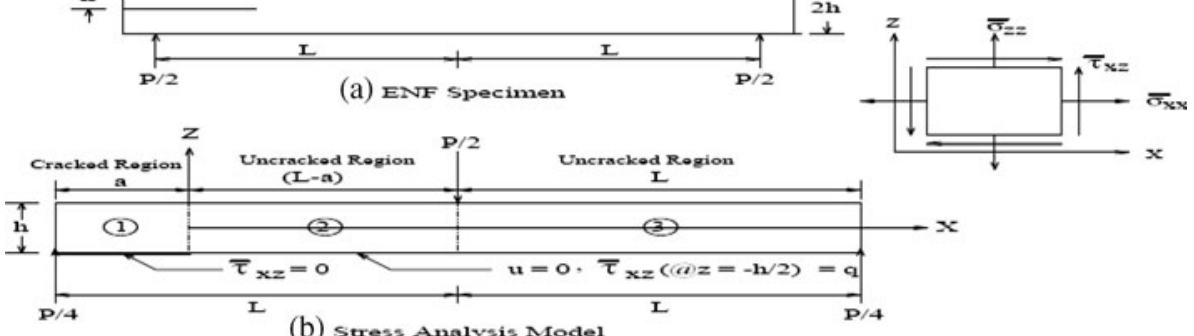

(b) Stress Analysis Model

Figure 2. ENF specimen and its stress analysis mode.

Raghu Prasad \& Pavankumar (2008), Pavankumar \& Raghu Prasad (2003, 2008, 2009), Pavankumar (2004). No credit is claimed for the variational method adopted to derive the governing differential equation and also the idea of matching conditions because they are exactly same as the method in reference mentioned above. This contribution is trying to obtain a more effective solution through the method developed in the present paper viz. MADM. Therefore, in order to prove the effectiveness of MADM the same examples with the same nomenclature in the reference are solved by MADM. Therefore, several equations like those for boundary conditions and matching conditions are repeated and they appear in references mentioned. The compliance and strain energy release rate (SERR), obtained from the present paper, have been compared with the existing experimental, analytical and finite element results in the literature. The unidirectional ENF and ENC specimens considered for the analysis have been shown in figures $2 \mathrm{a}$ and $3 \mathrm{a}$. The respective stress analysis models have been shown in figures $2 \mathrm{~b}$ and $3 \mathrm{~b}$ and these are similar to the stress analysis models proposed by Whitney (1990). These stress analysis models consider only upper halves i.e., above delamination plane of ENF and ENC specimens because of the fact that delamination is at mid-plane and lamination scheme is symmetric about the mid-plane of ENF and ENC specimens. The stress analysis models of ENF and ENC specimens have cracked and uncracked regions. In the uncracked region, at the bottom of the stress analysis models i.e., at the mid-plane of the actual ENF and ENC specimens, surface traction ' $q$ ' exists and axial displacement $u$ is zero. Further, it has been assumed that the delaminated faces slide over each other freely which means that the frictional effects between the delaminated faces have been neglected. The appropriate governing differential equations, for
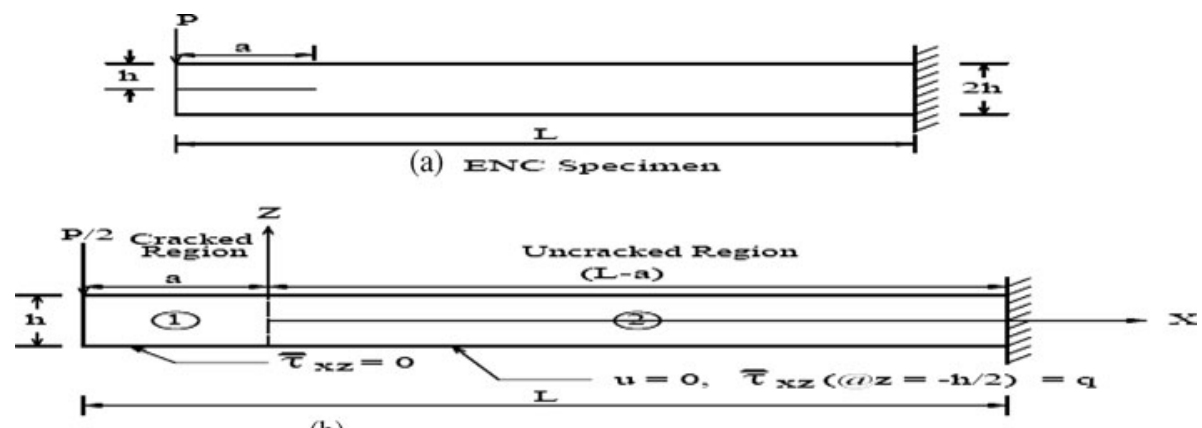

(b) Stress Amalysis Mrodel

Figure 3. ENC specimen and its stress analysis mode. 
cracked and uncracked regions of the ENF and ENC specimens, can be written based on FOBT and SOBT which have been derived using MADM. Then the governing differential equations of cracked and uncracked regions can be solved systematically. The solution consists of integration constants which can be determined from boundary and matching conditions. 'Special attention is necessary for continuity or matching conditions at the crack tip. In the present paper, appropriate matching conditions, in terms of generalized displacements and stress resultants, have been applied at the crack tip by enforcing displacement continuity at the crack tip in conjunction with variational equation' $(2003,2008,2009)$. Once the solution is obtained, deflection under the load can be determined using appropriate expressions. Further, compliance can be determined from the deflection under the load and strain energy release rate (SERR) can be calculated using MADM or numerical approach. It should be noted that the present solutions of ENF and ENC specimens are perfectly compatible with deformations that occur in the lower halves of the ENF and ENC specimens.

\subsection{Mathematical model}

Again, here the mathematical model is borrowed from the earlier work by Raghu Prasad \& Pavankumar (2008), Pavankumar \& Raghu Prasad (2003, 2008, 2009), Pavankumar (2004). In the stress analysis mode of ENF specimen, one can have two regions viz., cracked $[-a \leq x \leq$ $0]$ and uncracked $[0 \leq x \leq(2 L-a)]$ regions. The regions $[-a \leq x \leq 0],[0 \leq x \leq(L-$ $a)]$ and $[(L-a) \leq x \leq(2 L-a)]$ have been idealized as three different beams 1,2 and 3 , respectively with imaginary cuts at the crack tip and at the point of load application. To get appropriate governing differential equations, based on various laminated composite beam theories, for the analysis of cracked and uncracked regions of ENF and ENC specimens, the following substitutions need to be done in the equations.

\section{1a Cracked region:}

$$
\begin{aligned}
& \bar{\tau}_{x z}\left(\text { at } z= \pm \frac{h}{2}\right)=0 \\
& \bar{\sigma}_{z z}\left(\text { at } z= \pm \frac{h}{2}\right)=0 .
\end{aligned}
$$

2.1b Uncracked region:

$$
\begin{gathered}
\bar{\tau}_{x z}\left(\text { at } z=-\frac{h}{2}\right)=q \\
\bar{\tau}_{x z}\left(\text { at } z=\frac{h}{2}\right)=0 \\
\bar{\sigma}_{z z}\left(\text { at } z= \pm \frac{h}{2}\right)=0 .
\end{gathered}
$$

2.2 MADM procedure for ENF and ENC specimens using first order shear deformation beam theory

2.2a Governing differential equations for cracked and uncracked regions: The equilibrium equations for uncracked region, based on FOBT, can be obtained by using equation (2) as in the 
equations, and the equilibrium equations for uncracked region, based on FOBT, can be obtained by Raghu Prasad \& Pavankumar (2008), Pavankumar \& Raghu Prasad (2003, 2008, 2009), Pavankumar (2004) as

$$
\begin{aligned}
\frac{d N_{x x}}{d x}-b q & =0 \Rightarrow L_{x} N_{x x}-b q=0 \\
\frac{d Q_{x z}}{d x}=0 & \Rightarrow L_{x} Q_{x z}=0 \\
\frac{d M_{x x}}{d x}-Q_{x z}+\frac{b h}{2} q=0 & \Rightarrow L_{x} M_{x x}-Q_{x z}+\frac{b h}{2} q=0,
\end{aligned}
$$

where linear operator is $L_{x}=\frac{d}{d x}$. Further, for the cracked region, equilibrium equations can be obtained from the above equation (3) by putting $q=0$ i.e., delaminated faces slide freely over each other.

2.2b Inter-laminar shear stress resultant expressions for cracked and uncracked regions: It is possible to consider two choices of inter-laminar shear stress resultant expressions for cracked and uncracked regions and they are as follows, it may also be noted here that MADM solution for CBT is not obtained because $Q_{x z}$ becomes zero.

$F O B T^{1}$ : For both cracked and uncracked regions, inter-laminar shear stress resultants, have been considered with shear correction factor $k_{1}^{2}=1$. Choosing shear correction factor as unity is equivalent to saying that shear correction factor is not introduced into the formulation. This choice has been named as FOBT ${ }^{1}$.

$F O B T^{2}$ : For both cracked and uncracked regions, inter-laminar shear stress resultant, have been considered with the assumed shear correction factor $k_{1}^{2}=\frac{5}{6}$. This choice has been named as $\mathrm{FOBT}^{2}$.

Beside $\mathrm{SOBT}^{2}$ as explained above as one other solution classified as $\mathrm{SOBT}^{1}$ is available in the work of Raghu Prasad \& Pavankumar (2008), Pavankumar \& Raghu Prasad (2003, 2008, 2009), Pavankumar (2004). MADM solutions are obtained for SOBT ${ }^{1}$ with shear correction factors $k_{1}^{2}$. It may also be noted here that MADM solution for CBT is not obtained because $Q_{x z}$ becomes zero.

$$
\begin{gathered}
Q_{x z}=k_{1}^{2} G b h\left(\frac{d w_{\circ}}{d x}+\psi_{x}\right)+f_{q} b h q \\
=k_{1}^{2} G b h\left(L_{x} w_{\circ}+\psi_{x}\right)+f_{q} b h q
\end{gathered}
$$

and the quantities $k_{1}^{2}$ and $f_{q}$ will take the values as given in the following table depending upon cracked or uncracked regions. The MADM solution for $k_{1}^{2}=1$ and $\frac{5}{6}$ does not show much difference in $\mathrm{FOBT}^{1,2}$, therefore FOBT $^{E}$ is represented as FOBT. Similarly, FOBT and CBT MADM results are closure. For CBT $\psi_{x}=-L_{x} w_{\circ}=-\frac{d w_{\circ}}{d x}$ and the above equation (4) becomes $Q_{x z}=f_{q} b h q$.

2.2c MADM Solution: Cracked Region $[-a \leq x \leq 0$ ] - Beam 1 (figures $2 b$ and $3 b$ ):

$L_{x}^{-1}$ of first and second of equation (3) (with $q=0$ ) gives

$$
\begin{gathered}
L_{x} N_{x x}=0 \Rightarrow N_{x x}=C_{1} \\
L_{x} Q_{x z}=0 \Rightarrow Q_{x z}=C_{2},
\end{gathered}
$$


$L_{x}^{-1}$ of resulting equation, obtained from the substitution of equation (5) in the third of equation (3) (with $q=0$ ), gives

$$
L_{x} M_{x x}=Q_{x z} \Rightarrow M_{x x}=C_{2} x+C_{3} .
$$

By using stress resultant expressions in equation (4) in equations (1), (3) and (5) (with $q=0$ ), one can get the following equilibrium equations in terms of generalized displacements,

$$
\begin{gathered}
\bar{E} b h \frac{d u_{\circ}}{d x}=C_{1}, \Rightarrow \bar{E} b h L_{x} u_{\circ}=C_{1}, \Rightarrow u_{\circ}=C_{4}+L_{x}^{-1} \frac{C_{1}}{E b h} \\
G b h k_{1}^{2}\left(\frac{d w_{\circ}}{d x}+\psi_{x}\right)=C_{2}, \Rightarrow G b h k_{1}^{2}\left(L_{x} w_{\circ}+\psi_{x}\right)=C_{2}, \\
w_{\circ}=C_{5}+L_{x}^{-1} \frac{C_{2}}{G b h k_{1}^{2}}-L_{x}^{-1} \psi_{x} \\
\frac{\bar{E} b h^{3}}{12} \frac{d \psi_{x}}{d x}=C_{2} x+C_{3}, L_{x} \psi_{x}=\frac{C_{2} x+C_{3}}{E I} \\
\psi_{x}=C_{6}+L_{x}^{-1} \frac{12\left(C_{2} x+C_{3}\right)}{E b h^{3}} .
\end{gathered}
$$

From the above equations (5)-(7), one can re-write using MADM $u_{\circ}=C_{4}+L_{x}^{-1} \frac{C_{1}}{\bar{E} b h}$ and $\left(L_{x} w_{\circ}+\psi_{x}\right)=\frac{C_{2}}{G b h k_{1}^{2}}$ from this equation $\psi_{x}=\frac{C_{2}}{G b h k_{1}^{2}}-L_{x} w_{\circ}=C_{5}+\frac{6 C_{2} x^{2}+12 C_{3} x}{E b h^{3}}$ and using MADM one can find, $L_{x} w_{\circ}=\frac{C_{2}}{G b h k_{1}^{2}}-\left(C_{5}+\frac{6 C_{2} x^{2}+12 C_{3} x}{E b h^{3}}\right)$, it can be solved easily. The solution for cracked region $[-a \leq x \leq 0]$ (beam 1) can be summarized as

$$
\begin{gathered}
u_{\circ}=\frac{C_{1} x}{\bar{E}_{11} b h}+C_{4} \\
w_{\circ}=\frac{C_{2}}{\bar{E}_{11} b}\left(\frac{1}{k_{1}^{2}} \frac{\bar{E}_{11}}{G_{13}} \frac{x}{h}-2 \frac{x^{3}}{h^{3}}\right)-6 \frac{C_{3}}{\bar{E}_{11} b h} \frac{x^{2}}{h^{2}}-C_{5} h \frac{x}{h}+C_{6} \\
\psi_{x}=6 \frac{C_{2}}{\bar{E}_{11} b h} \frac{x^{2}}{h^{2}}+12 \frac{C_{3}}{\bar{E}_{11} b h^{2}} \frac{x}{h}+C_{5} .
\end{gathered}
$$

From these generalized displacement expressions, corresponding stress resultant expressions can be obtained easily by using the above equations.

Uncracked Region $(0 \leq x \leq(2 L-a))$ :

In the uncracked region i.e., beams 2 and $3, q \neq 0$. Further, axial displacement along $z=-\frac{h}{2}$ has been assumed to be zero. This can be written as $u\left(z=-\frac{h}{2}\right)=0$. By using the above equation (1), $u_{\circ}$ can be expressed in terms of $\psi_{x}$ as, $u_{\circ}=\frac{h}{2} \psi_{x}$ and now the surface traction ' $q$ ' is the unknown. Hence, in-plane stress resultant expression of $N_{x x}$ will be modified, by using the above relation,

$$
N_{x x}=\frac{\bar{E}_{11} b h^{2}}{2} \frac{d \psi_{x}}{d x}
$$


Further, $L_{x}^{-1}$ of second of equation (3) gives,

$$
Q_{x z}=\bar{C}_{1} \text {. }
$$

By substituting the modified in-plane stress resultant expression $N_{x x}$ as in the above equation (9), stress resultant expression of $M_{x x}$ and inter-laminar shear stress resultant expression of equation (10), first and third equations of equation (3), one can get the following equilibrium equations in terms of generalized displacements and surface traction ' $q$ ' for uncracked region.

$$
\begin{gathered}
\frac{d^{2} \psi_{x}}{d x^{2}}-\frac{2}{\bar{E}_{11} h^{2}} q=0 \Rightarrow L_{2 x} \psi_{x}-\frac{2}{\bar{E}_{11} h^{2}} q=0 \\
\left(\frac{d w_{\circ}}{d x}+\psi_{x}\right)+\frac{f_{q}}{G_{13} k_{1}^{2}} q=\frac{\bar{C}_{1}}{G_{13} k_{1}^{2} b h} \\
\Rightarrow\left(L_{x} w_{\circ}+\psi_{x}\right)+\frac{f_{q}}{G_{13} k_{1}^{2}} q=\frac{\bar{C}_{1}}{G_{13} k_{1}^{2} b h} \\
\frac{d^{2} \psi_{x}}{d x^{2}}+\frac{6 q}{\bar{E}_{11} h^{2}}=\frac{12 \bar{C}_{1}}{\bar{E}_{11} b h^{3}} \Rightarrow L_{2 x} \psi_{x}+\frac{6 q}{\bar{E}_{11} h^{2}}=\frac{12 \bar{C}_{1}}{\bar{E}_{11} b h^{3}},
\end{gathered}
$$

where linear operator $L_{2 x}=\frac{d^{2}}{d x^{2}}$. From the first of equations (11), one can re-write MADM form as, $Q_{x z}=C_{2}=G_{13} b h k_{1}^{2}\left(L_{x} w_{\circ}+\psi_{x}\right)$ or $\left(L_{x} w_{\circ}+\psi_{x}\right)=\frac{C_{2}}{G_{13} b h k_{1}^{2}}$, also can know the relation $N_{x x}=C_{1}+b q x=\bar{E}_{11} b h L_{x} u_{\circ}, u_{0}=\frac{b q \frac{x^{2}}{2}+C_{1} x}{\bar{E}_{11} b h}+C_{4}$ with known $u_{0}, \psi_{x}$ can be obtained as $\left(u_{0}\right.$ at $\left.\mathrm{x}=\frac{-h}{2}\right) \psi_{x}$ as $\psi_{x}=\left(C_{5}+\frac{-12 C_{1} x^{2}+6 C_{2} x^{2}+12 C_{3} x+24 C_{4} \bar{E}_{13} b x^{2}}{\bar{E}_{13} b h^{3}}\right)+\frac{f_{q}}{G_{13} k_{1}^{2}}$ and $\frac{d w_{0}}{d x}=\frac{C_{2}}{G_{13} b h K_{1}^{2}}-\psi_{x}+\frac{f_{q}}{G_{13} k_{1}^{2}}$ it can be solved easily. Substitution of the above equations in the second equation (11) yields

$$
w_{\circ}=\frac{\bar{C}_{1}}{\bar{E}_{11} b}\left\{\left(4 \frac{x^{2}}{h^{2}}-\frac{3}{2} f_{q}\right) \frac{\bar{E}_{11}}{G_{13}} \frac{1}{k_{1}^{2}} \frac{x}{h}-\frac{1}{2} \frac{x^{3}}{h^{3}}\right\}-\bar{C}_{2} \frac{x^{2}}{2}-\bar{C}_{3} x+\bar{C}_{4} .
$$

For the uncracked region $(0 \leq x \leq(L-a))$ (beam 2), solution can be summarized as

$$
\begin{gathered}
w_{\circ}=\frac{\bar{C}_{1}}{E_{11} b}\left\{\left(4 \frac{x^{2}}{h^{2}}-\frac{3}{2} f_{q}\right) \frac{\bar{E}_{11}}{G_{13}} \frac{1}{k_{1}^{2}}-\frac{1}{2} \frac{x^{3}}{h^{3}}\right\}-\bar{C}_{2} \frac{x^{2}}{2}-\bar{C}_{3} x+\bar{C}_{4} \\
q=\frac{4 C_{1}}{b h}-\frac{8 \bar{E} C_{4}}{h} .
\end{gathered}
$$

Similar solution can be obtained for uncracked region $((L-a) \leq x \leq(2 L-a)))$ (beam 3) and the solution can be written, with different integration constants as

$$
\begin{gathered}
w_{\circ}=\frac{\widetilde{C}_{1}}{\bar{E}_{11} b}\left\{\left(4 \frac{x^{2}}{h^{2}}-\frac{3}{2} f_{q}\right) \frac{\bar{E}_{11}}{G_{13}} \frac{1}{k_{1}^{2}} \frac{x}{h}-\frac{1}{2} \frac{x^{3}}{h^{3}}\right\}-\widetilde{C}_{2} \frac{x^{2}}{2}-\widetilde{C}_{3} x+\widetilde{C}_{4} \\
q=\frac{4 C_{1}}{b h}-\frac{8 \bar{E} C_{4}}{h} .
\end{gathered}
$$


From these generalized displacement expressions, corresponding stress resultant expressions can be obtained easily by using equations (14a-14b). According to FOBT, for ENF and ENC specimens, the unknown constants have to be determined from boundary and matching conditions given in the sections 2.2 and 2.3 for ENF and ENC specimens, respectively. Application of these boundary and matching conditions results in simultaneous equations for ENF and ENC specimens. These simultaneous equations have been solved using MADM for the unknown constants. Once these constants are determined, deflection under the load at $x=(L-a)$ for ENF specimen and at $x=-a$ for ENC specimen has been determined using equations for ENF and ENC specimens, respectively. From the deflection thus obtained, compliance and then SERR have been obtained using the procedures presented in section 3 .

\subsection{MADM Solution for ENF and ENC specimens using second order shear deformation beam theory}

2.3a Governing differential equations for cracked and uncracked regions: The equilibrium equations for uncracked region, based on SOBT, can be obtained as given below

$$
\begin{aligned}
\frac{d N_{x x}}{d x}-b q & =0 \Rightarrow L_{x} N_{x x}-b q=0 \\
\frac{d Q_{x z}}{d x} & =0 \Rightarrow L_{x} Q_{x z}=0 \\
\frac{d M_{x x}}{d x}-Q_{x z}+\frac{b h}{2} q & =0 \Rightarrow L_{x} M_{x x}-Q_{x z}+\frac{b h}{2} q=0 \\
\frac{d S_{x x}}{d x}-2 R_{x z}+\frac{b h^{2}}{4} q & =0 \Rightarrow L_{x} S_{x x}-2 R_{x z}-\frac{b h^{2}}{4} q=0 .
\end{aligned}
$$

Further, for the cracked region, equilibrium equations can be obtained from the above equation (15) by putting $q=0$ (i.e. delaminated faces slide freely over each other).

2.3b Inter-laminar shear stress resultants for cracked and uncracked regions: It is possible to consider two choices of inter-laminar shear stress resultant expressions for cracked and uncracked regions and they are as follows.

$S O B T^{1}$ : For both cracked and uncracked regions, inter-laminar shear stress resultants given by equations considered with shear correction factors $k_{1}^{2}=1$ and $k_{2}^{2}=1$. Choosing all shear correction factors as unity is equivalent to saying that shear correction factors are not introduced into the formulation. This choice has been named as $\mathrm{SOBT}^{1}$.

$S O B T^{2}$ : For both cracked and uncracked regions, inter-laminar shear stress resultants given by equations considered with shear correction factors $k_{1}^{2}=\frac{5}{6}$ and $k_{2}^{2}=\frac{7}{10}$. This choice has been named as $\mathrm{SOBT}^{2}$. Inter-laminar shear stress resultant expressions of $\mathrm{SOBT}^{E}, \mathrm{SOBT}^{1}$ and $\mathrm{SOBT}^{2}$ will be written in an unified form as,

$$
\begin{aligned}
& Q_{x z}=k_{1}^{2} G_{13} b h\left(L_{x} w_{\circ}+\psi_{x}\right)+f_{q} b h q \\
& R_{x z}=k_{2}^{2} G_{13} \frac{b h^{3}}{6} \phi_{x}-f_{r} b h^{2} q
\end{aligned}
$$


Table 1. Shear correction factors and other quantities for FOBT.

\begin{tabular}{cccccc}
\hline & \multicolumn{2}{c}{ Cracked region } & & \multicolumn{2}{c}{ Uncracked region } \\
\cline { 2 - 3 } \cline { 5 - 6 } & $k_{1}^{2}$ & $f_{q}$ & & $k_{1}^{2}$ & $f_{q}$ \\
\hline FOBT $^{E}$ & $5 / 6$ & 0 & & $5 / 6$ & $1 / 12$ \\
FOBT $^{1}$ & 1 & 0 & & 1 & 0 \\
FOBT $^{2}$ & $5 / 6$ & 0 & & $5 / 6$ & 0 \\
\hline
\end{tabular}

and the quantities $k_{1}^{2}, k_{2}^{2}, f_{q}$ and $f_{r}$ will take the values as given in tables 1 and 2 depending upon cracked or uncracked regions.

\section{3c MADM Solution:}

Cracked Region $[-a \leq x \leq 0]-$ Beam 1 (figures $2 b$ and $3 b)$ :

$L_{x}^{-1}$ of first and second of equation (15) (with $q=0$ ) gives

$$
\begin{gathered}
N_{x x}=C_{1} \\
Q_{x z}=C_{2},
\end{gathered}
$$

$L_{x}^{-1}$ of resulting equation, obtained from the substitution of equation (16) in the third of equation (15) (with $q=0$ ), gives

$$
M_{x x}=C_{2} x+C_{3} .
$$

By using stress resultant expressions in equation (5) substitute in equations (15)-(18) and in the last equation of equation (15) (with $q=0$ ), one can get the following equilibrium equations in terms of generalized displacements,

$$
\begin{gathered}
\bar{E}_{11} b h\left(L_{x} u_{\circ}+\frac{h^{2}}{24} L_{x} \phi_{x}\right)=C_{1} \\
G_{13} b h k_{1}^{2}\left(L_{x} w_{\circ}+\psi_{x}\right)=C_{2} \\
\frac{\bar{E}_{11} b h^{3}}{12} L_{x} \psi_{x}=C_{2} x+C_{3} \\
\frac{\bar{E}_{11} b h^{3}}{24}\left(L_{2 x} u_{\circ}+\frac{3 h^{2}}{40} L_{2 x} \phi_{x}\right) k_{2}^{2} G_{13} \frac{b h^{2}}{12} \phi_{x}=0 .
\end{gathered}
$$

Table 2. Shear correction factors and other quantities for SOBT.

\begin{tabular}{lccccccccc}
\hline & \multicolumn{4}{c}{ Cracked region } & & \multicolumn{4}{c}{ Uncracked region } \\
\cline { 2 - 4 } & $k_{1}^{2}$ & $k_{2}^{2}$ & $f_{q}$ & $f_{r}$ & & $k_{1}^{2}$ & $k_{2}^{2}$ & $f_{q}$ & $f_{r}$ \\
\hline SOBT $^{E}$ & $5 / 6$ & $7 / 10$ & 0 & 0 & & $5 / 6$ & $7 / 10$ & $1 / 12$ & $1 / 40$ \\
SOBT $^{1}$ & 1 & 1 & 0 & 0 & & 1 & 1 & 0 & 0 \\
SOBT $^{2}$ & $5 / 6$ & $7 / 10$ & 0 & 0 & & $5 / 6$ & $7 / 10$ & 0 & 0 \\
\hline
\end{tabular}


From the first of equation (20), one can write

$$
L_{x} u_{\circ}=\frac{C_{1}}{\bar{E}_{11} b h}-\frac{h^{2}}{24} L_{x} \phi_{x} .
$$

$L_{x}^{-1}$ of above equation gives,

$$
u_{\circ}=\frac{C_{1}}{\bar{E}_{11} b h} x-\frac{h^{2}}{24} \phi_{x}+C_{4} .
$$

Using equation (9) in the fourth of equation (20), one can get,

$$
\frac{d^{2} \phi_{x}}{d x^{2}}-\lambda_{1}^{2} h^{2} \phi_{x}=0 \Rightarrow L_{2 x} \phi_{x}=\lambda_{1}^{2} h^{2} \phi_{x},
$$

where, $\lambda_{1}=\sqrt{60 k_{2}^{2} \frac{G_{13}}{\bar{E}_{11}}}$. Using MADM procedure

$$
L_{2 x} \phi_{x}-\lambda_{1}^{2} h^{2} \phi_{x}=0 \Rightarrow L_{2 x} \phi_{x}=\lambda_{1}^{2} h^{2} \phi_{x} .
$$

Pre-multiplying both sides of the equation (23) by $L_{2 x}^{-1}$.

$$
\begin{gathered}
L_{2 x}^{-1} L_{2 x} \phi_{x}=L_{2 x}^{-1}\left(\lambda_{1}^{2} h^{2} \phi_{x}\right) \\
\phi_{x}=C_{5}+x C_{6}+L_{2 x}^{-1} \lambda_{1}^{2} h^{2} \phi_{x} \\
\phi_{x 0}=\phi_{x i}=C_{5}+x C_{6} \\
\phi_{x 1}=L_{2 x}^{-1}\left(\lambda_{1}^{2} h^{2}\right) \phi_{x 0}=\left(\lambda_{1}^{2} h^{2}\right)\left(C_{5} \frac{x^{2}}{2 !}+C_{6} \frac{x^{3}}{3 !}\right) \\
\phi_{x 2}=L_{2 x}^{-1}\left(\lambda_{1}^{2} h^{2}\right) \phi_{x 1}=\left(\lambda_{1}^{2} h^{2}\right)^{2}\left(C_{5} \frac{x^{4}}{4 !}+C_{6} \frac{x^{5}}{5 !}\right) \\
\vdots \\
\phi_{x n}=L_{2 x}^{-1}\left(\lambda_{1}^{2} h^{2}\right) \phi_{x_{n-1}}=\left(\lambda_{1}^{2} h^{2}\right)^{n}\left(C_{5} \frac{x^{2 n}}{2 n !}+C_{6} \frac{x^{2 n+1}}{(2 n+1) !}\right) .
\end{gathered}
$$

The MADM solution for equation (23) can be written as

$$
\begin{aligned}
\phi_{x}= & \sum_{i=0}^{n}=\phi_{x}^{i}=\phi_{x 0}+\phi_{x 1}+\phi_{x 2}+\ldots+\phi_{x_{n-1}}=C_{5}+x C_{6} \\
& +\left(\lambda_{1}^{2} h^{2}\right)\left(C_{5} \frac{x^{2}}{2 !}+C_{6} \frac{x^{3}}{3 !}\right)+\left(\lambda_{1}^{2} h^{2}\right)^{2}\left(C_{5} \frac{x^{4}}{4 !}+C_{6} \frac{x^{5}}{5 !}\right)+\ldots \\
= & C_{5}\left(1+\left(\lambda_{1}^{2} h^{2}\right) \frac{x^{2}}{2 !}+\left(\lambda_{1}^{2} h^{2}\right)^{2} \frac{x^{4}}{4 !}+\ldots+\left(\lambda_{1}^{2} h^{2}\right)^{n} \frac{x^{2 n}}{(2 n) !}\right) \\
& +C_{6}\left(x+\left(\lambda_{1}^{2} h^{2}\right) \frac{x^{3}}{3 !}+\left(\lambda_{1}^{2} h^{2}\right)^{2} \frac{x^{5}}{5 !}+\ldots+\left(\lambda_{1}^{2} h^{2}\right)^{n} \frac{x^{2 n+1}}{(2 n+1) !}\right) .
\end{aligned}
$$


Hence, by using equation (22) in equation (21), $u_{\circ}$ can be written as,

$$
\begin{aligned}
u_{\circ}= & C_{4}-\left(C_{5}\left(1+\left(\lambda_{1}^{2} h^{2}\right) \frac{x^{2}}{2 !}+\left(\lambda_{1}^{2} h^{2}\right)^{2} \frac{x^{4}}{4 !}+\ldots+\left(\lambda_{1}^{2} h^{2}\right)^{n} \frac{x^{2 n}}{(2 n) !}\right)\right. \\
& \left.+C_{6}\left(x+\left(\lambda_{1}^{2} h^{2}\right) \frac{x^{3}}{3 !}+\left(\lambda_{1}^{2} h^{2}\right)^{2} \frac{x^{5}}{5 !}+\ldots+\left(\lambda_{1}^{2} h^{2}\right)^{n} \frac{x^{2 n+1}}{(2 n+1) !}\right)\right)+\frac{C_{1}}{\bar{E}_{11} b h} .
\end{aligned}
$$

From the third of equation (20), one can have

$$
L_{x} \psi_{x}=\frac{12}{\bar{E}_{11} b h^{3}} C_{2} x+\frac{12}{\bar{E}_{11} b h^{3}} C_{3}
$$

$L_{x}^{-1}$ of above equation gives,

$$
\psi_{x}=\frac{6}{\bar{E}_{11} b h^{3}} C_{2} x^{2}+\frac{12}{\bar{E}_{11} b h^{3}} C_{3} x+C_{7} .
$$

Second of equations (20) can be written as,

$$
\left(L_{x} w_{\circ}+\psi_{x}\right)=\frac{C_{2}}{k_{1}^{2} G_{13} b h} .
$$

The resulting equation of $L_{x}^{-1}$ of above equation can be written as,

$$
w_{\circ}=\frac{C_{2} x}{k_{1}^{2} G_{13} b h}-L_{x}^{-1} \psi_{x} d x+C_{8}=\frac{C_{2} x}{k_{1}^{2} G_{13} b h}-\int_{0}^{x} \psi_{x} d x+C_{8} .
$$

By using equation (25) in equation (27), one can get

$$
w_{\circ}=\frac{C_{2}}{\bar{E}_{11} b}\left(\frac{1}{k_{1}^{2}} \frac{\bar{E}_{11}}{G_{13}} \frac{x}{h}-2 \frac{x^{3}}{h^{3}}\right)-6 \frac{C_{3}}{\bar{E}_{11} b h} \frac{x^{2}}{h^{2}}-C_{7} h \frac{x}{h}+C_{8} .
$$

The MADM solution for cracked region $[-a \leq x \leq 0]$ (beam 1) can be summarized as,

$$
\begin{gathered}
u_{\circ}=C_{4}\left(C_{5}\left(1+\left(\lambda_{1}^{2} h^{2}\right) \frac{x^{2}}{2 !}+\left(\lambda_{1}^{2} h^{2}\right)^{2} \frac{x^{4}}{4 !}+\ldots+\left(\lambda_{1}^{2} h^{2}\right)^{n} \frac{x^{2 n}}{(2 n) !}\right)+\frac{C_{1}}{\bar{E}_{11} b h}\right. \\
\left.+C_{6}\left(x+\left(\lambda_{1}^{2} h^{2}\right) \frac{x^{3}}{3 !}+\left(\lambda_{1}^{2} h^{2}\right)^{2} \frac{x^{5}}{5 !}+\ldots+\left(\lambda_{1}^{2} h^{2}\right)^{n} \frac{x^{2 n+1}}{(2 n+1) !}\right)\right) \\
w_{\circ}=\frac{C_{2}}{\bar{E}_{11} b}\left(\frac{1}{k_{1}^{2}} \frac{\bar{E}_{11}}{G_{13}} \frac{x}{h}-2 \frac{x^{3}}{h^{3}}\right)-6 \frac{C_{3}}{E_{11} b h} \frac{x^{2}}{h^{2}}-C_{7} h \frac{x}{h}+C_{8} \\
\phi_{x}=\left(C_{5}\left(1+\left(\lambda_{1}^{2} h^{2}\right) \frac{x^{2}}{2 !}+\left(\lambda_{1}^{2} h^{2}\right)^{2} \frac{x^{4}}{4 !}+\ldots+\left(\lambda_{1}^{2} h^{2}\right)^{n} \frac{x^{2 n}}{(2 n) !}\right)\right. \\
\left.+C_{6}\left(x+\left(\lambda_{1}^{2} h^{2}\right) \frac{x^{3}}{3 !}+\left(\lambda_{1}^{2} h^{2}\right)^{2} \frac{x^{5}}{5 !}+\ldots+\left(\lambda_{1}^{2} h^{2}\right)^{n} \frac{x^{2 n+1}}{(2 n+1) !}\right)\right) \\
\psi_{x}=6 \frac{C_{2}}{\bar{E}_{11} b h} \frac{x^{2}}{h^{2}}+12 \frac{C_{3}}{\bar{E}_{11} b h^{2}} \frac{x}{h}+C_{7} .
\end{gathered}
$$


From these generalized displacement expressions, corresponding stress resultant expressions can be obtained easily by using equations (31a-31d).

Uncracked region $[0 \leq x \leq(2 L-a)]-$ Beams 2 and 3 (figures $2 b$ and $3 b$ ):

In the uncracked region i.e., beams 2 and $3, q \neq 0$. Further, axial displacement along $z=-\frac{h}{2}$ has been assumed to be zero $\left(z=-\frac{h}{2}\right.$ is the bottom surface of the stress analysis mode shown in figures $2 b$ and $3 b$. This can be written as,

$$
u\left(z=-\frac{h}{2}\right)=0
$$

By using the above equation (22), $u_{\circ}$ can be expressed in terms of $\psi_{x}$ and $\phi_{x}$ as

$$
u_{\circ}=\frac{h}{2} \psi_{x}-\frac{h^{2}}{4} \phi_{x}
$$

and now the surface traction ' $q$ ' is the unknown. Hence, in-plane stress resultant expressions of $N_{x x}$ and $S_{x x}$ will be modified, by using equation (20) as,

$$
\begin{aligned}
& N_{x x}=\frac{\bar{E}_{11} b h^{2}}{2}\left(\frac{d \psi_{x}}{d x}-\frac{h}{3} \frac{d \phi_{x}}{d x}\right)=\frac{\bar{E}_{11} b h^{2}}{2}\left(L_{x} \psi_{x}-\frac{h}{3} L_{x} \phi_{x}\right) \\
& S_{x x}=\frac{\bar{E}_{11} b h^{4}}{24}\left(\frac{d \psi_{x}}{d x}-\frac{h}{5} \frac{d \phi_{x}}{d x}\right)=\frac{\bar{E}_{11} b h^{4}}{24}\left(L_{x} \psi_{x}-\frac{h}{5} L_{x} \phi_{x}\right) .
\end{aligned}
$$

Further, $L_{x}^{-1}$ of second of equation (15) gives,

$$
Q_{x z}=\bar{C}_{1}
$$

By using the two modified in-plane stress resultant expressions $N_{x x}, S_{x x}$ in equation (34a, b), stress resultant expression of $M_{x x}$ and inter-laminar shear stress resultant expressions in the equation (35), first and last two equations of equation (15), one can get the following equilibrium equations in terms of generalized displacements and surface traction ' $q$ ' for uncracked region.

$$
\begin{gathered}
\frac{d^{2} \psi_{x}}{d x^{2}}-\frac{h}{3} \frac{d^{2} \phi_{x}}{d x^{2}}-\frac{2}{\bar{E}_{11} h^{2}} q=L_{2 x} \psi_{x} \frac{h}{3} L_{2 x} \phi_{x}-\frac{2}{\bar{E}_{11} h^{2}} q=0 \\
\left(\frac{d w_{\circ}}{d x}+\psi_{x}\right)+\frac{f_{q}}{G_{13} k_{1}^{2}} q=\left(L_{x} w_{\circ}+\psi_{x}\right)+\frac{f_{q}}{G_{13} k_{1}^{2}} q=\frac{\bar{C}_{1}}{G_{13} k_{1}^{2} b h} \\
L_{2 x} \psi_{x}-\frac{12}{h^{2}} \frac{G_{13}}{\bar{E}_{11}}\left\{k_{1}^{2}\left(L_{x} w_{\circ}+\psi_{x}\right)\right\} \frac{12 f_{q} q}{\bar{E}_{11} h^{2}}+\frac{6 q}{\bar{E}_{11} h^{2}}=0 \\
L_{2 x} \psi_{x}-\frac{h}{5} L_{2 x} \phi_{x}-\frac{8 k_{2}^{2}}{h} \frac{\bar{E}_{11}}{G_{13}} \phi_{x}+\frac{2\left(24 f_{r}-3\right)}{\bar{E}_{11} h^{2}} q=0 .
\end{gathered}
$$

From the first of equation (36a-36d), one can write

$$
q=\frac{\bar{E}_{11} h^{2}}{2}\left\{\frac{d^{2} \psi_{x}}{d x^{2}}-\frac{h}{3} \frac{d^{2} \phi_{x}}{d x^{2}}\right\}=\frac{\bar{E}_{11} h^{2}}{2}\left\{L_{2 x} \psi_{x}-\frac{h}{3} L_{2 x} \phi_{x}\right\} .
$$


Using the second of equation (36a-36d) in the third of equation (36a-36d), one can get

$$
\frac{d^{2} \psi_{x}}{d x^{2}}-\frac{12}{h^{2}} \frac{\bar{C}_{1}}{\bar{E}_{11} b h}+\frac{6}{\bar{E}_{11} h^{2}} q=L_{2 x} \psi_{x}-\frac{12}{h^{2}} \frac{\bar{C}_{1}}{\bar{E}_{11} b h}+\frac{6}{\bar{E}_{11} h^{2}} q=0
$$

Substitution of equation (37) in equation (36) gives

$$
L_{2 x} \psi_{x}=\frac{h}{4} L_{2 x} \phi_{x}+\frac{3}{h^{2}} \frac{\bar{C}_{1}}{\bar{E}_{11} b h}
$$

$L_{x}^{-1}$ of equation (39) twice gives,

$$
\psi_{x}=\frac{h}{4} \phi_{x}+\frac{3}{2} \frac{\bar{C}_{1} x^{2}}{\bar{E}_{11} b h^{3}}+\bar{C}_{2} x+\bar{C}_{3}
$$

Expression for $q$ can be rewritten, by using equation (39) in equation (37), as

$$
q=-\frac{\bar{E}_{11} h^{3}}{24} L_{2 x} \phi_{x}+\frac{3}{2} \frac{\bar{C}_{1}}{b h} .
$$

From the second of equation (36a-36d), one can have

$$
\left(L_{x} w_{\circ}+\psi_{x}\right)=-\frac{f_{q}}{G_{13} k_{1}^{2}} q+\frac{\bar{C}_{1}}{G_{13} k_{1}^{2} b h} .
$$

By multiplying $L_{x}^{-1}$ the above equation (27) and rearranging, one can get,

$$
\begin{aligned}
w_{\circ} & =-L_{x}^{-1} \psi_{x} d x-\frac{f_{q}}{G_{13} k_{1}^{2}} L_{x}^{-1} q d x+\frac{\bar{C}_{1} x}{G_{13} k_{1}^{2} b h}+\bar{C}_{4} \\
& =-\int_{0}^{x} \psi_{x} d x-\frac{f_{q}}{G_{13} k_{1}^{2}} \int_{0}^{x} q d x+\frac{\bar{C}_{1} x}{G_{13} k_{1}^{2} b h}+\bar{C}_{4} .
\end{aligned}
$$

Substitution of equations (24) and (26) in the fourth of equation (36) results in the following equation:

$$
L_{2 x} \phi_{x}-\frac{\lambda_{2}^{2}}{h^{2}} \phi_{x}=\frac{\bar{S}_{1}}{\bar{E}_{11} b h^{2}} \bar{C}_{1}
$$

where $\lambda_{2}=\sqrt{\frac{80 k_{2}^{2}}{\left(3-20 f_{r}\right)} \frac{G_{13}}{\bar{E}_{11}}}, \bar{S}_{1}=-\frac{30\left(24 f_{r}-2\right)}{\left(3-20 f_{r}\right)}$.

The MADM solution for equation (43) can be written as

$$
L_{2 x} \phi_{x}=\frac{\lambda_{2}^{2}}{h^{2}} \phi_{x}+\frac{\bar{S}_{1}}{\bar{E}_{11} b h^{2}} \bar{C}_{1} .
$$


Pre-multiplying both sides of the equation (43) by $L_{2 x}^{-1}$.

$$
\begin{aligned}
& L_{2 x}^{-1} L_{2 x} \phi_{x}=L_{2 x}^{-1}\left(\frac{\lambda_{2}^{2}}{h^{2}} \phi_{x}+\frac{\bar{S}_{1}}{\bar{E}_{11} b h^{2}} \bar{C}_{1}\right) \\
& \phi_{x}=\bar{C}_{5}+x \bar{C}_{6}+L_{2 x}^{-1}\left(\frac{\lambda_{2}^{2}}{h^{2}} \phi_{x}+\frac{\bar{S}_{1}}{\bar{E}_{11} b h^{2}} \bar{C}_{1}\right) \\
& \phi_{x i}=\bar{C}_{5}+x \bar{C}_{6}+L_{2 x}^{-1}\left(\frac{\bar{S}_{1}}{\bar{E}_{11} b h^{2}} \bar{C}_{1}\right)=\bar{C}_{5}+x \bar{C}_{6}+\left(\frac{\bar{S}_{1}}{\bar{E}_{11} b h^{2}} \bar{C}_{1}\right) \frac{x^{2}}{2 !} \\
& \phi_{x 0}=\bar{C}_{5}+x \bar{C}_{6} \\
& \phi_{x 1}=L_{2 x}^{-1}\left(\frac{\bar{S}_{1}}{\bar{E}_{11} b h^{2}} \bar{C}_{1}\right)+L_{2 x}^{-1}\left(\frac{\lambda_{2}^{2}}{h^{2}} \phi_{x 0}\right) \\
& =\frac{\bar{S}_{1}}{\bar{E}_{11} b h^{2}} \bar{C}_{1} \frac{x^{2}}{2 !}+\left(\frac{\lambda_{2}^{2}}{h^{2}}\right)\left(\bar{C}_{5} \frac{x^{2}}{2 !}+\bar{C}_{6} \frac{x^{3}}{3 !}\right) \\
& \phi_{x 2}=L_{2 x}^{-1}\left(\frac{\lambda_{2}^{2}}{h^{2}} \phi_{x 1}\right)=\left(\frac{\lambda_{2}^{2}}{h^{2}}\right)^{2}\left(\bar{C}_{5} \frac{x^{4}}{4 !}+\bar{C}_{6} \frac{x^{5}}{5 !}\right)+\left(\frac{\lambda_{2}^{2}}{h^{2}}\right)\left(\frac{\bar{S}_{1}}{\bar{E}_{11} b h^{2}} \bar{C}_{1} \frac{x^{6}}{6 !}\right) \\
& \phi_{x n}=L_{2 x}^{-1}\left(\frac{\lambda_{2}^{2}}{h^{2}} \phi_{x n-1}\right)=\left(\frac{\lambda_{2}^{2}}{h^{2}}\right)^{n}\left(\bar{C}_{5} \frac{x^{2 n}}{2 n !}+\bar{C}_{6} \frac{x^{2 n+1}}{2 n+1 !}\right)+\left(\frac{\lambda_{2}^{2}}{h^{2}}\right)^{n-1}\left(\frac{\bar{S}_{1}}{\bar{E}_{11} b h^{2}} \bar{C}_{1} \frac{x^{2(n+1)}}{2(n+1) !}\right) .
\end{aligned}
$$

Hence, the MADM solution for equation (43) can be written as

$$
\begin{aligned}
\phi_{x}= & \bar{C}_{5}\left(1+\left(\frac{\lambda_{2}^{2}}{h^{2}}\right) \frac{x^{2}}{2 !}+\left(\frac{\lambda_{2}^{2}}{h^{2}}\right)^{2} \frac{x^{4}}{4 !}+\left(\frac{\lambda_{2}^{2}}{h^{2}}\right)^{3} \frac{x^{6}}{6 !}+\ldots+\left(\frac{\lambda_{2}^{2}}{h^{2}}\right)^{n} \frac{x^{2 n}}{(2 n) !}\right) \\
& +\bar{C}_{6}\left(x+\left(\frac{\lambda_{2}^{2}}{h^{2}}\right) \frac{x^{3}}{3 !}+\left(\frac{\lambda_{2}^{2}}{h^{2}}\right)^{2} \frac{x^{5}}{5 !}+\left(\frac{\lambda_{2}^{2}}{h^{2}}\right)^{3} \frac{x^{7}}{7 !}+\ldots+\left(\frac{\lambda_{2}^{2}}{h^{2}}\right)^{n} \frac{x^{2 n+1}}{(2 n+1) !}\right) \\
+ & \frac{\bar{S}_{1}}{\bar{E}_{11} b h^{2}} \bar{C}_{1}\left(\frac{x^{2}}{2 !}+\left(\frac{\lambda_{2}^{2}}{h^{2}}\right) \frac{x^{4}}{4 !}+\left(\frac{\lambda_{2}^{2}}{h^{2}}\right)^{2} \frac{x^{6}}{6 !}+\left(\frac{\lambda_{2}^{2}}{h^{2}}\right)^{3} \frac{x^{8}}{8 !}\right. \\
& \left.+\ldots+\left(\frac{\lambda_{2}^{2}}{h^{2}}\right)^{n-1} \frac{x^{2(n+1)}}{(2(n+1)) !}\right) .
\end{aligned}
$$

Substitution of equation (45) in the equation (40) gives

$$
\psi_{x}=\frac{\bar{C}_{1}}{\bar{E}_{11} b h}\left(\frac{3}{2} \frac{x^{2}}{h^{2}}\right)+\bar{C}_{2} x+\bar{C}_{3}+\phi_{x} .
$$


Using equation (45) in the equation (41), one can get,

$$
\begin{aligned}
q= & -\frac{\bar{E}_{11} h^{3}}{24} L_{2 x}\left(\bar{C}_{5}\left(1+\left(\frac{\lambda_{2}^{2}}{h^{2}}\right) \frac{x^{2}}{2 !}+\left(\frac{\lambda_{2}^{2}}{h^{2}}\right)^{2} \frac{x^{4}}{4 !}+\left(\frac{\lambda_{2}^{2}}{h^{2}}\right)^{3} \frac{x^{6}}{6 !}+\ldots+\left(\frac{\lambda_{2}^{2}}{h^{2}}\right)^{n} \frac{x^{2 n}}{(2 n) !}\right)\right. \\
& +\bar{C}_{6}\left(x+\left(\frac{\lambda_{2}^{2}}{h^{2}}\right) \frac{x^{3}}{3 !}+\left(\frac{\lambda_{2}^{2}}{h^{2}}\right)^{2} \frac{x^{5}}{5 !}+\left(\frac{\lambda_{2}^{2}}{h^{2}}\right)^{3} \frac{x^{7}}{7 !}+\ldots+\left(\frac{\lambda_{2}^{2}}{h^{2}}\right)^{n} \frac{x^{2 n+1}}{(2 n+1) !}\right)+\frac{3}{2} \frac{\bar{C}_{1}}{b h} \\
& +\frac{\bar{S}_{1}}{\bar{E}_{11} b h^{2}} \bar{C}_{1}\left(\frac{x^{2}}{2 !}+\left(\frac{\lambda_{2}^{2}}{h^{2}}\right) \frac{x^{4}}{4 !}+\left(\frac{\lambda_{2}^{2}}{h^{2}}\right)^{2} \frac{x^{6}}{6 !}+\left(\frac{\lambda_{2}^{2}}{h^{2}}\right)^{3} \frac{x^{8}}{8 !}\right. \\
& \left.+\ldots+\left(\frac{\lambda_{2}^{2}}{h^{2}}\right)^{n-1} \frac{x^{2(n+1)}}{(2(n+1)) !}\right) .
\end{aligned}
$$

Substitution of equation (45), (46) in the equation (43) gives,

$$
\begin{aligned}
w_{\circ}= & \frac{\bar{C}_{1}}{\bar{E}_{11} b}\left\{\bar{S}_{2} \frac{x}{h}-\frac{1}{2} \frac{x^{3}}{h^{3}}\right\}-\bar{C}_{2} \frac{x^{2}}{2}-\bar{C}_{3} x+\bar{C}_{4} \\
& +h^{2} \bar{S}_{3} \bar{C}_{5}\left(1+\left(\frac{\lambda_{2}^{2}}{h^{2}}\right) \frac{x^{2}}{2 !}+\left(\frac{\lambda_{2}^{2}}{h^{2}}\right)^{2} \frac{x^{4}}{4 !}+\left(\frac{\lambda_{2}^{2}}{h^{2}}\right)^{3} \frac{x^{6}}{6 !}+\ldots+\left(\frac{\lambda_{2}^{2}}{h^{2}}\right)^{n} \frac{x^{2 n}}{(2 n) !}\right) \\
& -h^{2} \bar{S}_{3} \bar{C}_{6}\left(x+\left(\frac{\lambda_{2}^{2}}{h^{2}}\right) \frac{x^{3}}{3 !}+\left(\frac{\lambda_{2}^{2}}{h^{2}}\right)^{2} \frac{x^{5}}{5 !}+\left(\frac{\lambda_{2}^{2}}{h^{2}}\right)^{3} \frac{x^{7}}{7 !}+\ldots+\left(\frac{\lambda_{2}^{2}}{h^{2}}\right)^{n} \frac{x^{2 n+1}}{(2 n+1) !}\right) \\
& +h^{2} \bar{S}_{3} \frac{\bar{S}_{1}}{\bar{E}_{11} b h^{2}} \bar{C}_{1}\left(\frac{x^{2}}{2 !}+\left(\frac{\lambda_{2}^{2}}{h^{2}}\right) \frac{x^{4}}{4 !}+\left(\frac{\lambda_{2}^{2}}{h^{2}}\right)^{2} \frac{x^{6}}{6 !}+\left(\frac{\lambda_{2}^{2}}{h^{2}}\right)^{3} \frac{x^{8}}{8 !}\right. \\
& \left.+\ldots+\left(\frac{\lambda_{2}^{2}}{h^{2}}\right)^{n-1} \frac{x^{2(n+1)}}{(2(n+1)) !}\right),
\end{aligned}
$$

where $\bar{S}_{2}=\left(1-\frac{3}{2} f_{q}\right) \frac{\bar{E}_{11}}{G_{13}} \frac{1}{k_{1}^{2}}+\frac{\bar{S}_{1}}{4 \lambda_{2}^{2}}$,

$$
\bar{S}_{3}=\lambda_{2}^{2}\left(\frac{f_{q}}{24 k_{1}^{2}} \frac{\bar{E}_{11}}{G_{13}}-\frac{1}{4 \lambda_{2}^{2}}\right) .
$$

For the uncracked region $[0 \leq x \leq(L-a)]$ (beam 2), solution can be summarized as

$$
\begin{aligned}
w_{\circ}= & \frac{\bar{C}_{1}}{\bar{E}_{11} b}\left\{\bar{S}_{2} \frac{x}{h}-\frac{1}{2} \frac{x^{3}}{h^{3}}\right\}-\bar{C}_{2} \frac{x^{2}}{2}-\bar{C}_{3} x+\bar{C}_{4}+\bar{C}_{5} h^{2} \bar{S}_{3} \\
& \left(1+\left(\frac{\lambda_{2}^{2}}{h^{2}}\right) \frac{x^{2}}{2 !}+\left(\frac{\lambda_{2}^{2}}{h^{2}}\right)^{2} \frac{x^{4}}{4 !}+\left(\frac{\lambda_{2}^{2}}{h^{2}}\right)^{3} \frac{x^{6}}{6 !}+\ldots+\left(\frac{\lambda_{2}^{2}}{h^{2}}\right)^{n} \frac{x^{2 n}}{(2 n) !}\right) \\
& -\bar{C}_{6} h^{2} \bar{S}_{3}\left(x+\left(\frac{\lambda_{2}^{2}}{h^{2}}\right) \frac{x^{3}}{3 !}+\left(\frac{\lambda_{2}^{2}}{h^{2}}\right)^{2} \frac{x^{5}}{5 !}+\ldots+\left(\frac{\lambda_{2}^{2}}{h^{2}}\right)^{n} \frac{x^{2 n+1}}{(2 n+1) !}\right)
\end{aligned}
$$




$$
\begin{aligned}
& \psi_{x}=\frac{\bar{C}_{1}}{\bar{E}_{11} b h}\left(\frac{3}{2} \frac{x^{2}}{h^{2}}-\frac{\bar{S}_{1}}{4 \lambda_{2}^{2}}\right)+\bar{C}_{2} x+\bar{C}_{3}+\bar{C}_{5} \frac{h}{4} \\
& \left(1+\left(\frac{\lambda_{2}^{2}}{h^{2}}\right) \frac{x^{2}}{2 !}+\left(\frac{\lambda_{2}^{2}}{h^{2}}\right)^{2} \frac{x^{4}}{4 !}+\left(\frac{\lambda_{2}^{2}}{h^{2}}\right)^{3} \frac{x^{6}}{6 !}+\ldots+\left(\frac{\lambda_{2}^{2}}{h^{2}}\right)^{n} \frac{x^{2 n}}{(2 n) !}\right) \\
& +\bar{C}_{6} \frac{h}{4}\left(x+\left(\frac{\lambda_{2}^{2}}{h^{2}}\right) \frac{x^{3}}{3 !}+\left(\frac{\lambda_{2}^{2}}{h^{2}}\right)^{2} \frac{x^{5}}{5 !}+\ldots+\left(\frac{\lambda_{2}^{2}}{h^{2}}\right)^{n} \frac{x^{2 n+1}}{(2 n+1) !}\right) \\
& \phi_{x}=\frac{\bar{S}_{1}}{\bar{E}_{11} b h^{2}} \bar{C}_{1}\left(\frac{x^{2}}{2 !}+\left(\frac{\lambda_{2}^{2}}{h^{2}}\right) \frac{x^{4}}{4 !}+\left(\frac{\lambda_{2}^{2}}{h^{2}}\right)^{2} \frac{x^{6}}{6 !}+\ldots+\left(\frac{\lambda_{2}^{2}}{h^{2}}\right)^{n-1} \frac{x^{2(n+1)}}{(2(n+1)) !}\right) \\
& +\bar{C}_{5}\left(1+\left(\frac{\lambda_{2}^{2}}{h^{2}}\right) \frac{x^{2}}{2 !}+\left(\frac{\lambda_{2}^{2}}{h^{2}}\right)^{2} \frac{x^{4}}{4 !}+\left(\frac{\lambda_{2}^{2}}{h^{2}}\right)^{3} \frac{x^{6}}{6 !}+\ldots+\left(\frac{\lambda_{2}^{2}}{h^{2}}\right)^{n} \frac{x^{2 n}}{(2 n) !}\right) \\
& +\bar{C}_{6}\left(x+\left(\frac{\lambda_{2}^{2}}{h^{2}}\right) \frac{x^{3}}{3 !}+\left(\frac{\lambda_{2}^{2}}{h^{2}}\right)^{2} \frac{x^{5}}{5 !}+\ldots+\left(\frac{\lambda_{2}^{2}}{h^{2}}\right)^{n} \frac{x^{2 n+1}}{(2 n+1) !}\right) \\
& q=\frac{3}{2} \frac{\bar{C}_{1}}{b h}-\frac{\bar{E}_{11} \lambda_{2}^{2} h}{24}\left(\bar{C}_{5}\left(1+\left(\frac{\lambda_{2}^{2}}{h^{2}}\right) \frac{x^{2}}{2 !}+\left(\frac{\lambda_{2}^{2}}{h^{2}}\right)^{2} \frac{x^{4}}{4 !}+\ldots+\left(\frac{\lambda_{2}^{2}}{h^{2}}\right)^{n} \frac{x^{2 n}}{(2 n) !}\right)\right. \\
& \left.+\bar{C}_{6}\left(x+\left(\frac{\lambda_{2}^{2}}{h^{2}}\right) \frac{x^{3}}{3 !}+\left(\frac{\lambda_{2}^{2}}{h^{2}}\right)^{2} \frac{x^{5}}{5 !}+\ldots+\left(\frac{\lambda_{2}^{2}}{h^{2}}\right)^{n} \frac{x^{2 n+1}}{(2 n+1) !}\right)\right) \text {. }
\end{aligned}
$$

Similar solution can be obtained for uncracked region $[(L-a) \leq x \leq(2 L-a)]$ (beam 3$)$ and the solution can be written, with different integration constants as

$$
\begin{aligned}
& w_{\circ}= \frac{\widetilde{C}_{1}}{\widetilde{E}_{11} b}\left\{\widetilde{S}_{2} \frac{x}{h}-\frac{1}{2} \frac{x^{3}}{h^{3}}\right\}-\widetilde{C}_{2} \frac{x^{2}}{2}-\widetilde{C}_{3} x+\widetilde{C}_{4}+\widetilde{C}_{5} h^{2} \widetilde{S}_{3} \\
&\left(1+\left(\frac{\lambda_{2}^{2}}{h^{2}}\right) \frac{x^{2}}{2 !}+\left(\frac{\lambda_{2}^{2}}{h^{2}}\right)^{2} \frac{x^{4}}{4 !}+\left(\frac{\lambda_{2}^{2}}{h^{2}}\right)^{3} \frac{x^{6}}{6 !}+\ldots+\left(\frac{\lambda_{2}^{2}}{h^{2}}\right)^{n} \frac{x^{2 n}}{(2 n) !}\right) \\
&-\widetilde{C}_{6} h^{2} \widetilde{S}_{3}\left(x+\left(\frac{\lambda_{2}^{2}}{h^{2}}\right) \frac{x^{3}}{3 !}+\left(\frac{\lambda_{2}^{2}}{h^{2}}\right)^{2} \frac{x^{5}}{5 !}+\ldots+\left(\frac{\lambda_{2}^{2}}{h^{2}}\right)^{n} \frac{x^{2 n+1}}{(2 n+1) !}\right) \\
&\left(\begin{array}{c}
\left.\frac{\lambda_{2}^{2}}{h^{2}}\right) \frac{x^{2}}{2 !}+\left(\frac{\lambda_{2}^{2}}{h^{2}}\right)^{2} \frac{x^{4}}{4 !}+\left(\frac{\lambda_{2}^{2}}{h^{2}}\right)^{3} \frac{x^{6}}{6 !}+\ldots+\left(\frac{3}{\widetilde{E}_{11} b h} \frac{x_{2}^{2}}{h^{2}}-\frac{\widetilde{S}_{1}}{4 \lambda_{2}^{2}}\right\}+\widetilde{C}_{2} x+\widetilde{C}_{3}+\widetilde{C}_{5} \frac{h}{4} \\
(2 n) !
\end{array}\right)+ \\
& \widetilde{C}_{6} \frac{h}{4}\left(x+\left(\frac{\lambda_{2}^{2}}{h^{2}}\right)^{\frac{x^{3}}{3 !}}+\left(\frac{\lambda_{2}^{2}}{h^{2}}\right)^{\frac{x^{5}}{5 !}}+\ldots+\left(\frac{\lambda_{2}^{2}}{h^{2}}\right)^{n} \frac{x^{2 n+1}}{(2 n+1) !}\right)
\end{aligned}
$$




$$
\begin{aligned}
& \phi_{x}= \frac{\widetilde{S}_{1}}{\widetilde{E}_{11} b h^{2}} \widetilde{C}_{1}\left(\frac{x^{2}}{2 !}+\left(\frac{\lambda_{2}^{2}}{h^{2}}\right)^{2} \frac{x^{4}}{4 !}+\left(\frac{\lambda_{2}^{2}}{h^{2}}\right)^{2} \frac{x^{6}}{6 !}+\ldots+\left(\frac{\lambda_{2}^{2}}{h^{2}}\right)^{2} \frac{x^{2 n+1}}{(2(n+1)) !}\right) \\
&+\widetilde{C}_{5}\left(1+\left(\frac{\lambda_{2}^{2}}{h^{2}}\right) \frac{x^{2}}{2 !}+\left(\frac{\lambda_{2}^{2}}{h^{2}}\right)^{2} \frac{x^{4}}{4 !}+\left(\frac{\lambda_{2}^{2}}{h^{2}}\right)^{3} \frac{x^{6}}{6 !}+\ldots+\left(\frac{\lambda_{2}^{2}}{h^{2}}\right)^{n} \frac{x^{2 n}}{(2 n) !}\right) \\
&+\widetilde{C}_{6}\left(x+\left(\frac{\lambda_{2}^{2}}{h^{2}}\right) \frac{x^{3}}{3 !}+\left(\frac{\lambda_{2}^{2}}{h^{2}}\right)^{2} \frac{x^{5}}{5 !}+\ldots+\left(\frac{\lambda_{2}^{2}}{h^{2}}\right)^{n} \frac{x^{2 n+1}}{(2 n+1) !}\right) \\
& q=\frac{3}{2} \frac{\widetilde{C}_{1}}{b h}-\frac{\widetilde{E}_{11} \lambda_{2}^{2} h}{24}\left(\widetilde{C}_{5}\left(1+\left(\frac{\lambda_{2}^{2}}{h^{2}}\right) \frac{x^{2}}{2 !}+\left(\frac{\lambda_{2}^{2}}{h^{2}}\right)^{2} \frac{x^{4}}{4 !}+\ldots+\left(\frac{\lambda_{2}^{2}}{h^{2}}\right)^{n} \frac{x^{2 n}}{(2 n) !}\right)\right. \\
&\left.+\widetilde{C}_{6}\left(x+\left(\frac{\lambda_{2}^{2}}{h^{2}}\right) \frac{x^{3}}{3 !}+\left(\frac{\lambda_{2}^{2}}{h^{2}}\right)^{2} \frac{x^{5}}{5 !}+\ldots+\left(\frac{\lambda_{2}^{2}}{h^{2}}\right)^{n} \frac{x^{2 n+1}}{(2 n+1) !}\right)\right) .
\end{aligned}
$$

From these generalized displacement expressions, corresponding stress resultant expressions can be obtained easily by using equations (34) and (16). According to SOBT, the unknown constants (for ENF and ENC specimens) will have to be determined from boundary and matching conditions given in the sections 2.2 and 2.3 for ENF and ENC specimens, respectively. These simultaneous equations have been solved using MADM for the unknown constants. Once these constants are determined, deflection under the load (at $x=(L-a)$ for ENF specimen and at $x=-a$ for ENC specimen) has been determined using equations (32) and (18) for ENF and ENC specimens, respectively. From the deflection thus obtained, compliance and then SERR have been obtained using the procedures presented in section 3 .

\section{Determination of compliance and strain energy release rate}

The compliance ' $C$ ' can be obtained from the following relation $C=\frac{\delta}{P}$ in which ' $\delta$ ' is the deflection under the load $P$. In the case of ENF specimen, ' $\delta$ ' is obtained from $\delta=w_{\circ}^{(2)}$ [at $x=$ $(L-a)]$ or $w_{\circ}^{(3)}[$ at $x=(L-a)]$. Similarly, in the case of ENC specimen, ' $\delta$ ' is obtained from $\delta=w_{\circ}^{(1)}[$ at $x=-a]$. The SERR and the compliance are related by the following formula $G_{I I}=\frac{P^{2}}{2 b} \frac{d C}{d a}$. In the present paper, $\frac{d C}{d a}$ has not been determined explicitly as it is tedious. And hence, the derivative $\frac{d C}{d a}$ has been evaluated using MADM spatial discretization. Hence, one can obtain SERR from the following expression $G_{I I}=\frac{P^{2}}{2 b}\left(\frac{C_{n}-C_{n-1}}{\Delta}\right)=\frac{P^{2}}{2 b}\left(\frac{C_{n+1}-C_{n}}{\Delta}\right)$ (figure 4).

\section{Numerical examples}

In this section, first to validate present formulation, the compliance and the SERR, obtained from the analysis of unidirectional ENF and ENC specimens and using present formulation considering various theories FOBT, $\mathrm{SOBT}^{1,2}$ can be compared with the available results mentioned in Carlsson et al (1986a), Whitney (1990), Whitney et al (1987), Salpekar et al (1988), Sela et al (1989), Pavankumar \& Raghu Prasad (2003), Chatterjee (1991), Wang \& Williams (1992) in the literature. Further, parametric study can be carried out to study the influence of crack length(a), 


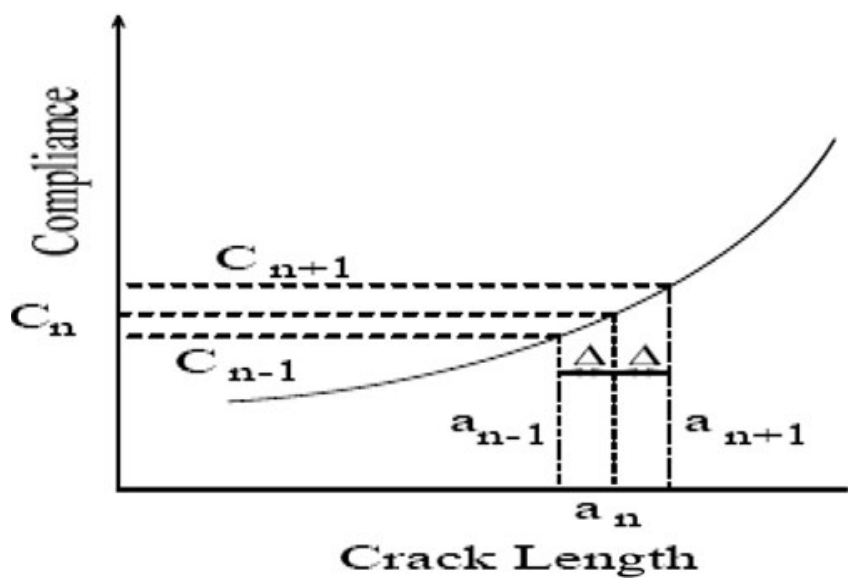

Figure 4. Numerical and compliance approach.

span to depth $\left(\frac{L}{h}\right)$ ratio and ratio of Young's modulus to shear modulus (shear deformation) $\left(\frac{E_{11}}{G_{13}}\right)$ on the compliance, SERR and shear stress distribution ahead of the crack tip. Also, comparative study can be made among the various laminated composite beam theories considered to bring out the importance of shear deformation theories i.e., FOBT and SOBT, clearly and particularly in the case of SERR.

\subsection{Geometrical, material properties and load for ENC and ENF problems}

Table 3 gives the material and geometrical properties as given by Sela et al (1989).

MADM Results: The results obtained from the present work have been compared with the experimental results in table 4. Comparative study has been made with the results of Wang \& Williams (1992) and for this purpose material and geometrical properties and load have been

Table 3. Material properties and geometrical properties from Sela et al (1989).

\begin{tabular}{c}
\hline Material Properties: $:^{\dagger}$ \\
$E_{11}=1.4 \times 10^{5}, E_{22}=1 \times 10^{4}$ \\
$G_{13}=6 \times 10^{3}$ \\
$v_{13}=0.34$ \\
\hline Geometrical Properties: \\
$L=$ half span $=50.8$ \\
$b=$ width 25.4 \\
$h=$ half thickness $=1.524$ \\
No. of laminae $=24, \mathrm{~N}=$ Lamina thickness $=0.127$ \\
$a=$ crack length $=25.4$ \\
$P_{c}=$ Load $=672.9$
\end{tabular}

${ }^{\dagger}$ All the dimensions are mentioned in $\mathrm{N}, \mathrm{mm}$ by default 
Table 4. Comparison of compliance and SERR from MADM with experimental results of Sela et al (1989), and analytical from Raghu Prasad \& Pavankumar (2008).

\begin{tabular}{lcccc}
\hline $\begin{array}{l}\text { Mode } \\
\text { Sela et al (1989) }\end{array}$ & \multicolumn{2}{c}{$\begin{array}{c}\text { Compliance } \\
0.0032\end{array}$} & \multicolumn{2}{c}{$\begin{array}{c}\text { SERR } G_{I I c} \frac{\mathrm{J}}{\mathrm{m}^{2}} \\
527 \\
\end{array}$} \\
\hline FOBT & 0.0031 & 494.7750 & 0.00312 & 516.4701 \\
SOBT $^{1}$ & 0.00315 & 494.775 & 0.00319 & 513.461 \\
SOBT $^{2}$ & 0.00316 & 494.775 & 0.00321 & 517.150 \\
SOBT $^{E}$ & 0.00318 & 495.468 & 0.00321 & 528.375 \\
\hline
\end{tabular}

given in the tables where the results have been presented in tables 4, 5, 6, 7, 8 and 9. Compliance and SERR values obtained from the present work have been compared with the experimentally obtained compliance and SERR values of Sela et al (1989). These comparisons have been presented in table 5. By closely examining table 5, it can be concluded that, among FOBT and $\mathrm{SOBT}^{1,2, E}$ beam theories considered, compliance and SERR values obtained from $\mathrm{SOBT}^{E}$ are in good agreement with the experimental and analytical obtained compliance and SERR values. The present work results have also been compared with those of Wang \& Williams (1992) for both ENF and ENC (also called as End Loaded Split (ELS) specimen) specimens and are presented in the tables 5, 6, 7, 8 and 9. Once again it can be observed that results from SOBT are in better agreement with those from experimental and FEM values of Wang \& Williams (1992) when compared to those of SOBT and also compared analytical results too. Tables 7 and 9 also reveal the fact as to how important is the SOBT deformation in calculating the SERR accurately. Having established the point that one can use plane stress type of analysis, in the further presentation plane stress type analysis results have been considered and also it is due to the reason that the width of the specimen is larger than its thickness. Further, parametric study can be carried out only for unidirectional ENF specimen because the conclusions that could be drawn

Table 5. Comparison of compliance and SERR from MADM with Wang \& Williams (1992) \& Raghu Prasad \& Pavankumar (2008) results for ENF specimen.

\begin{tabular}{|c|c|c|c|c|c|c|}
\hline \multicolumn{7}{|c|}{$\begin{array}{c}\text { Material }^{1}: S_{11}=S_{22}=7.69 \times 10^{-6}, S_{66}=2.5 \times 10^{-4}, v_{12}=0.3 \\
\text { Geometry: } L=50, b=1.0, h=1.5, P=1.0\end{array}$} \\
\hline & \multicolumn{2}{|c|}{ Wang \& Williams (1992) } & \multirow[t]{2}{*}{ Raghu Prasad \& Pavankumar (2008) } & \multicolumn{3}{|c|}{ MADM } \\
\hline$a$ & & & & $\mathrm{SOBT}^{1}$ & SOBT $^{2}$ & $\mathrm{SOBT}^{E}$ \\
\hline 10 & & 0.075 & 0.075 & 0.074 & 0.075 & 0.075 \\
\hline 20 & & 0.082 & 0.082 & 0.081 & 0.082 & 0.081 \\
\hline 30 & & 0.100 & 0.099 & 0.097 & 0.100 & 0.098 \\
\hline \multirow[t]{3}{*}{40} & & 0.134 & 0.133 & 0.128 & 0.133 & 0.131 \\
\hline & & & $\mathrm{SERR} \times 10^{3}\left(\frac{\mathrm{N}-\mathrm{mm}}{\mathrm{mm}^{2}}\right)$ & & & \\
\hline & FEM & Semi-empirical & & & & \\
\hline 10 & 0.163 & 0.160 & 0.130 & 0.146 & 0.157 & 0.155 \\
\hline 20 & 0.597 & 0.573 & 0.514 & 0.546 & 0.570 & 0.565 \\
\hline 30 & 1.293 & 1.243 & 1.155 & 1.203 & 1.238 & 1.231 \\
\hline 40 & 2.230 & 2.170 & 2.052 & 2.116 & 2.161 & 2.152 \\
\hline
\end{tabular}


Table 6. Comparison of compliance and SERR from MADM with Wang \& Williams (1992) and Raghu Prasad \& Pavankumar (2008) results for ENF specimen.

\begin{tabular}{|c|c|c|c|c|c|c|}
\hline \multicolumn{7}{|c|}{$\begin{array}{l}\text { Material }^{2}: S_{11}=6.8 \times 10^{-6}, S_{22}=128 \times 10^{-3}, S_{66}=362 \times 10^{-3} \\
\text { Geometry }^{2} \text { : Geometry, Poisson's ratio and Load same as material }\end{array}$} \\
\hline & \multicolumn{2}{|c|}{ Wang \& Williams (1992) } & \multirow[t]{2}{*}{ Raghu Prasad \& Pavankumar (2008) } & \multicolumn{3}{|c|}{ MADM } \\
\hline$a$ & & & & $\mathrm{SOBT}^{1}$ & $\mathrm{SOBT}^{2}$ & $\mathrm{SOBT}^{E}$ \\
\hline 10 & & 0.0676 & 0.0669 & 0.0676 & 0.0681 & 0.0689 \\
\hline 20 & & 0.0738 & 0.0723 & 0.0735 & 0.0744 & 0.0752 \\
\hline 30 & & 0.0896 & 0.0867 & 0.0888 & 0.0903 & 0.0914 \\
\hline \multirow[t]{3}{*}{40} & & 0.1197 & 0.1219 & 0.1181 & 0.1205 & 0.1147 \\
\hline & & & $\operatorname{SERR} \times 10^{3}\left(\frac{\mathrm{N}-\mathrm{mm}}{\mathrm{mm}^{2}}\right)$ & & & \\
\hline & FEM & Semi-empirical & & & & \\
\hline 10 & 0.151 & 0.151 & 0.144 & 0.134 & 0.147 & 0.116 \\
\hline 20 & 0.551 & 0.526 & 0.513 & 0.492 & 0.518 & 0.456 \\
\hline 30 & 1.182 & 1.128 & 1.108 & 1.077 & 1.116 & 1.022 \\
\hline 40 & 2.025 & 1.957 & 1.927 & 1.887 & 1.936 & 1.816 \\
\hline
\end{tabular}

for unidirectional ENF specimen could be valid for unidirectional ENC specimen also. Values of compliance, SERR and shear stress distribution ahead of the crack tip based on SOBT only could be presented in the plots in tables for the SOBT category. And, it should be noted that FOBT and $\mathrm{SOBT}^{1}$ can show the same trend as $\mathrm{SOBT}^{2, E}$, respectively with minor differences in the magnitudes of the results.

4.1a Influence of crack length $\left(\frac{a}{L}\right)$ ratio: The above tables show the variation of compliance and SERR, obtained from shear deformation theories. From these tables, it is observed that compliance and SERR increase as crack length increases. Compliance and SERR given by shear deformation theories are more than those from FOBT which reflects the importance of first

Table 7. Comparison of SERR from MADM with Wang \& Williams (1992) and Raghu Prasad \& Pavankumar (2008) results for ENF specimen.

\begin{tabular}{|c|c|c|c|c|c|c|}
\hline \multicolumn{7}{|c|}{$\begin{array}{l}\text { Material }^{3} \text { : Material properties, Poisson's ratio and load same as material } \\
\text { Geometry }\end{array}$} \\
\hline \multirow[t]{3}{*}{$S_{66}$} & \multicolumn{2}{|c|}{ Wang \& Williams (1992) } & \multirow[t]{3}{*}{ Raghu Prasad \& Pavankumar (2008) } & \multicolumn{3}{|c|}{ MADM } \\
\hline & & & & $\overline{\text { SOBT }^{1}}$ & SOBT $^{2}$ & SOBT $^{E}$ \\
\hline & & & & & & \\
\hline & FEM & Semi-empirical & & & & \\
\hline $181 \times 10^{-6}$ & 0.527 & 0.508 & 0.455 & 0.480 & 0.499 & 0.495 \\
\hline $362 \times 10^{-6}$ & 0.551 & 0.526 & 0.456 & 0.492 & 0.518 & 0.513 \\
\hline $724 \times 10^{-6}$ & 0.587 & 0.552 & 0.458 & 0.510 & 0.546 & 0.538 \\
\hline $145 \times 10^{-5}$ & 0.637 & 0.592 & 0.462 & 0.536 & 0.587 & 0.575 \\
\hline $500 \times 10^{-5}$ & 0.783 & 0.719 & 0.485 & 0.614 & 0.692 & 0.682 \\
\hline
\end{tabular}


Table 8. Comparison of compliance and SERR from MADM with Wang \& Williams (1992) and Raghu Prasad \& Pavankumar (2008) results for ENC specimen.

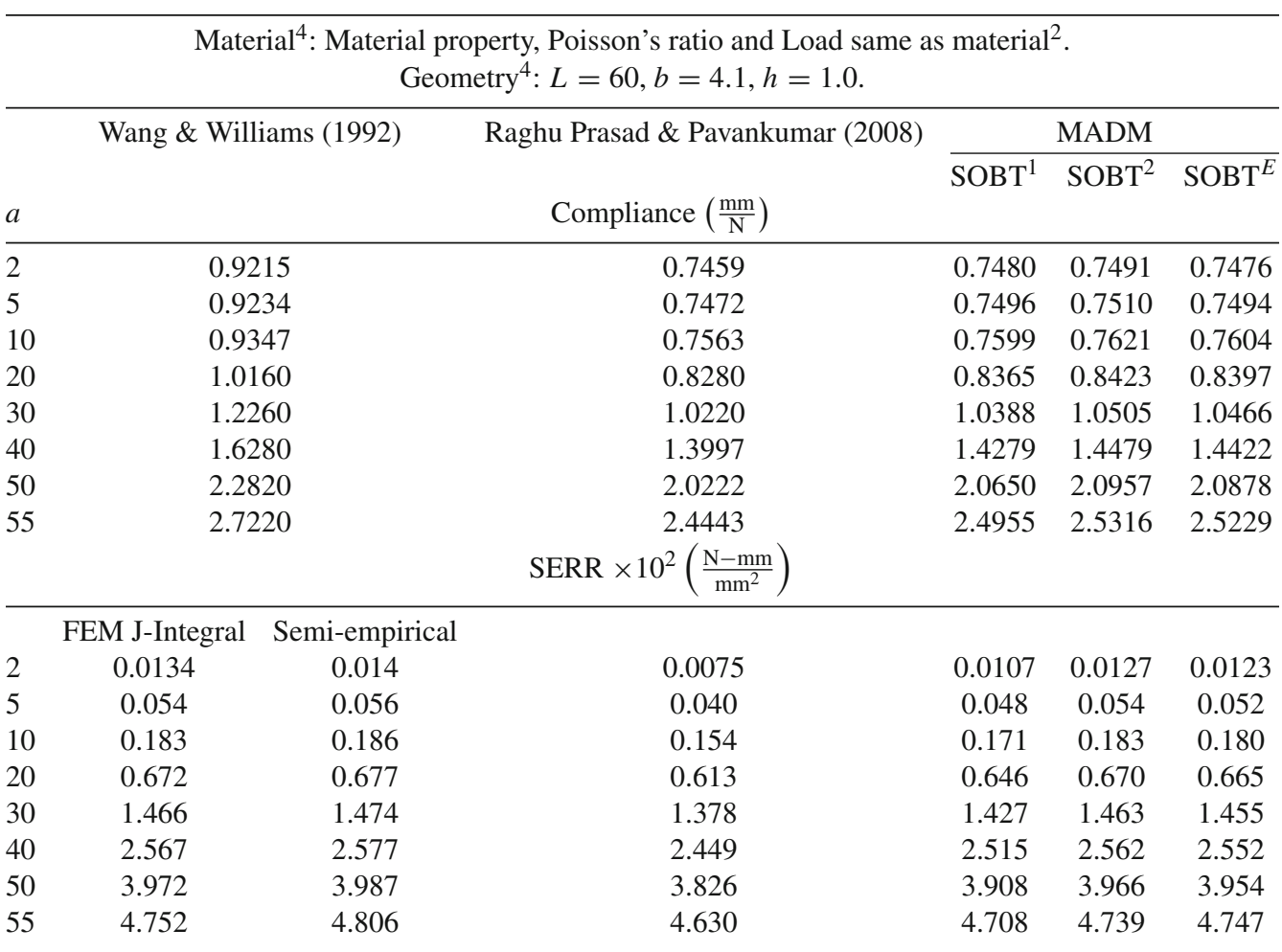

order shear deformation. Also tables show that the influence of crack length on the normalized compliance and SERR values obtained from the shear deformation theories. From table, it can be observed that normalized compliance values from $\mathrm{SOBT}^{1}$ show descending trend which

Table 9. Comparison of SERR from MADM with Wang \& Williams (1992) and Raghu Prasad \& Pavankumar (2008) results for ENC specimen.

Material $^{5}$ : Material property, Poisson's ratio and load same as material ${ }^{2}$.

Geometry ${ }^{5}$ : Geometry property same as geometry ${ }^{2}$.

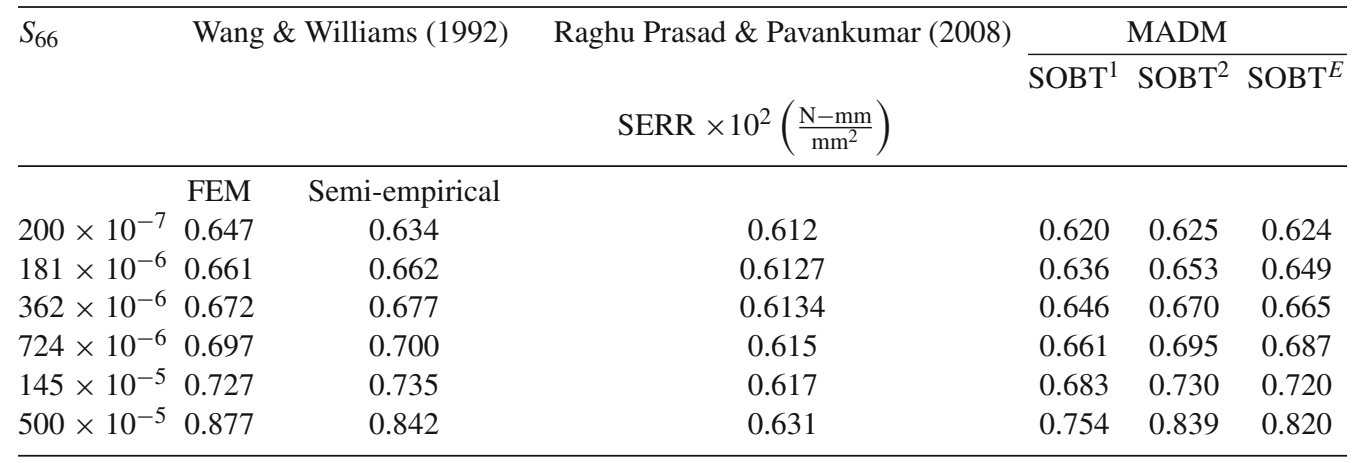


means that the theory $\mathrm{SOBT}^{E}$ tend towards FOBT as crack length increases. Normalized compliances from SOBT show ascending trend up to certain crack lengths and then afterwards show a descending trend based on the material property under consideration. In other words, based on the material properties, the difference between the compliance values from FOBT and SOBT theories increases up to a certain crack length and then afterwards decreases. The SERR values of $\mathrm{SOBT}^{E}$ approach those of FOBT as the crack length increases which can be seen clearly. The shear stress distribution ahead of the crack tip obtained from FOBT and SOBT beam theories for any $\frac{a}{L}$ (given crack length) is the shear stress uniform throughout i.e., constant. It could be seen that shear stress given by FOBT and $\mathrm{SOBT}^{1,2}$ is constant ahead of the crack tip. It implies that FOBT does not affect the shear stress distribution in the case of $\mathrm{SOBT}^{E}$ (i.e., independent of the shear deformation). Peak shear stress values of $\mathrm{SOBT}^{E}$ are slightly greater than those of FOBT.

\section{Results and discussion}

In this section, mode II fracture of one-dimensional layered laminate is analysed by MADM. The basic governing differential equations of equilibrium and matching conditions are borrowed from earlier work by Raghu Prasad \& Pavankumar (2008), Pavankumar \& Raghu Prasad (2003, 2008, 2009), Pavankumar (2004). While familiar methods already known to solve governing differential equations were employed by the earlier researchers as mentioned above. MADM is employed here. It is very interesting to see that MADM gives results which are closer to the experimental than the earlier methods (classical) as mentioned in Salpekar et al (1988), Sela et al (1989), Gillespie et al (1986), Wang \& Williams (1992), Chatterjee (1991), Whitney et al (1987), Whitney (1990), Corleto \& Hogan (1995), Carlsson et al (1986a).

It is evident that the results from MADM are much closer to the experimental than the results due to analytical methods employed by earlier researchers mentioned above. The reason is quite obvious. In the earlier works mentioned above, after obtaining the compliance $C$, the derivative $\frac{d C}{d a}$ was obtained by finite difference scheme, while in the present work it was not required. Not only compliance values $C$ were obtained by MADM, even $\frac{d C}{d a}$ values were obtained by MADM, which means errors arising due to the use of numerical schemes such as finite difference do not appear here. Here MADM values are represented in FOBT, SOBT ${ }^{1,2}$ and analytical values are represented in TOBT. Table 10 shows the values of compliance $(C)$ and SERR $G_{I I}$

Table 10. Difference in values between bench mark and MADM results.

\begin{tabular}{|c|c|c|c|c|c|c|c|}
\hline \multicolumn{8}{|c|}{ Results } \\
\hline \multirow[t]{2}{*}{ Table No: } & \multirow[t]{2}{*}{ Bench Mark } & \multirow[t]{2}{*}{ Parameter } & \multicolumn{4}{|c|}{ MADM } & \multirow[t]{2}{*}{ Analytical BKR et al } \\
\hline & & & $\mathrm{FOBT}^{E}$ & SOBT $^{1}$ & $\mathrm{SOBT}^{2}$ & $\mathrm{SOBT}^{E}$ & \\
\hline 4 & Expt. & Compl. & 0.0008 & 0.00001 & -0.00001 & -0.00001 & 0.00002 \\
\hline \multirow[t]{2}{*}{5} & Expt. & Compl. & & 0.001 & 0 & 0 & 0 \\
\hline & FEM & SERR & & 0.0170 & 0.0060 & 0.0080 & 0.0330 \\
\hline \multirow[t]{2}{*}{6} & Expt. & Compl. & & 0 & -0.0005 & -0.0013 & 0.0007 \\
\hline & FEM & SERR & & 0.0170 & 0.0040 & 0.0350 & 0.0070 \\
\hline 7 & FEM & SERR & & 0.047 & 0.028 & 0.032 & 0.072 \\
\hline \multirow[t]{2}{*}{8} & Expt. & Compl. & & 0.1756 & 0.1735 & 0.1739 & 0.1743 \\
\hline & FEM & SERR & & 0.0027 & 0.0007 & 0.0011 & 0.0059 \\
\hline 9 & FEM & SERR & & 0.027 & 0.022 & 0.023 & 0.035 \\
\hline
\end{tabular}


as obtained by Wang \& Williams (1992), Gillespie et al (1986), Carlsson et al (1986a), Whitney et al (1987), Whitney (1990) from experiments by Raghu Prasad \& Pavankumar (2008), Pavankumar \& Raghu Prasad (2003, 2008, 2009), Pavankumar (2004) by classical methods and by MADM in the current work. It is encouraging to note that MADM results are close and agree with the exact as well as analytical in trend.

The results show that the compliance $(C)$ and SERR $G_{I I}$ values are obtained by MADM are closer to the experimental ones by Salpekar et al (1988) than those of Pavankumar and Raghu Prasad as mentioned above, while the latter have adopted third order shear deformation theory. In other words, using MADM it is possible to get results closer to the experimental one without resorting to the higher order theories.

\section{Conclusions}

Mathematical models for the stress analysis of unidirectional ENF and ENC specimens using FOBT and SOBT have been developed and presented for inter-laminar fracture toughness of unidirectional composites in mode II. For this purpose, the available stress analysis models (Whitney 1990) for ENF and ENC specimens have been considered. These models consider only upper halves of ENF and ENC specimens because the delamination is at mid-plane and lamination scheme is symmetric about the mid-plane of ENF and ENC specimens. In the present paper, appropriate matching conditions, in terms of generalized displacements and stress resultants, have been applied at the crack tip by enforcing the displacement continuity at the crack tip in conjunction with variational equation. SERR has been calculated using compliance approach. The compliance and SERR obtained from the present formulations have been compared with the existing experimental, analytical and finite element results, it is observed that MADM is in good agreement with the existing results. Also, parametric study has been carried out to study the influence of crack length, ratio of Young's modulus to shear modulus and span to depth ratio of the specimen on the compliance and SERR. The following are some conclusions that have been drawn from the comparative and parametric studies using MADM (i.e., FOBT, $\mathrm{SOBT}^{1,2}$ ) for the different cases.

(i) Results from SOBT ${ }^{1,2}$ are closer to the results of Salpekar et al (1988), Sela et al (1989) and Chatterjee (1991) while results of SOBT and FOBT are relatively less close.

(ii) Compliance and SERR increase as crack length increases for all beam theories.

(iii) The SERR values from $\mathrm{SOBT}^{2}$ theory approach SERR values from FOBT as the crack length increases.

(iv) As $\frac{L}{h}$ ratio increases, compliance and SERR increase for FOBT \& $\mathrm{SOBT}^{1,2}$ beam theories.

The governing differential equations, for the SOBT \& FOBT, have been derived for unidirectional laminated composites using principle of MADM and variational principles. Griffith's crack growth criterion and compliance approach have been presented to determine the SERR.

\section{References}

Abbasbandy S 2003 Improving Newton-Raphson method for nonlinear equations by modified Adomian decomposition method. Appl. Math. Comput. 145(2): 887-893

Adomian G 1984a A new approach to nonlinear partial differential equations. J. Math. Anal. Appl. 102: 73-85 
Adomian G 1984b Convergent series solutions of nonlinear equations. Computer and Application in Mathematic 11(2): 225-230

Adomian G 1989a Nonlinear stochastic systems and applications to physics. Kluwer Publisher

Adomian G 1989b Nonlinear stochastic systems theory and application to physics. Dordrecht: Kluwer Academic

Adomian G 1994a Solution of physical problems by decomposition. Comput. Math. Appl. 27: 145-154

Adomian G 1994b Solving frontier problems of physics: The decomposition method (2nd edn). Dordrecht: Kluwer

Adomian G and Rach R 1996 Modified adomian polynomials. Math. Comput. Model. 24(11): 39-46

Adomian G, Rach R and Meyers R E 1991 An efficient methodology for the physical sciences. Kybenetes 20(7): 24-34

Barrett J D and Foschi R O 1977 Mode II stress intensity factors for cracked wood beams. Eng. Fract. Mech. 9(3): 371-378

Carlsson L A and Gillespie Jr J W 1989 Application of Fracture Mechanics to Composite Materials, chapter Mode II inter-laminar Fracture of Composites. Netherlands: Elsevier Science Publishers 6(3): 113-157

Carlsson L A, Gillespie Jr J W and Pipes R B 1986a On the analysis and design of End Notch Flexure (ENF) for Mode II Testing. J. Compos. Mater. 20(6): 594-604

Carlsson L A, Gillespie Jr J W and Whitney J M 1986b Inter-laminar fracture mechanics analysis of the end notched flexure specimen. In Proceedings of 1st Conference on Composite Materials, Lancaster, Technomic Publishing 9: 421-433

Chatterjee S N 1991 Analysis of test specimens for inter-laminar Mode II fracture toughness part 1 elastic laminates. J. Compos. Mater. 20(5): 470-493

Corleto C R and Hogan H A 1995 Energy release rates for the ENF specimen using a beam on an elastic foundation. J. Compos. Mater. 29(11): 1420-1436

Davidson B D, Kruger R and Konig M 1995 Three dimensional analysis and resulting design recommendations for uni-directional and multi-directional end notched flexure tests. J. Compos. Mater. 29(16): $2108-2133$

Davidson B D, Kruger R and Konig M 1996 Effect of stacking sequence on energy release rate distributions in multi-directional DCB and ENF specimens. Eng. Fract. Mech. 55(4): 557-569

Gillespie Jr J W, Carlsson L A and Pipes R B 1986 Finite element analysis of the end notch flexure specimen for measuring mode II fracture toughness. Compos. Sci. Technol. 27: 177-197

Pavankumar D V T G 2004 Analysis of composite end notch flexure specimen using various plate theories. NAL 1: 10-18

Pavankumar D V T G and Raghu Prasad B K 2003 Higher-order beam theories for mode II fracture of unidirectional composites. J. Appl. Mech., Trans. ASME 70(6): 840-852

Pavankumar D V T G and Raghu Prasad B K 2008 Analysis of unidirectional $\left(0^{\circ}\right)$ fiber-reinforced laminated composite double cantilever beam specimen using higher order beam theories. Eng. Fract. Mech. 75(8): 2156-2174

Pavankumar D V T G and Raghu Prasad B K 2009 Fracture behavior of multi-directional DCB specimen: Higher-order beam theories. J. Eng. Mech. 135(10): 1119-1128

Raghu Prasad B K and Pavankumar D V T G 2008 Analysis of composite ENF specimen using higher order beam theories. Thin-walled Struct. 46: 676-688

Russell A J and Street K N 1982 Factors affecting the inter-laminar fracture energy of graphitelepoxy laminates, Progress in Science and Engineering of Composites, ICCM-IV. In Kawata K, Hayashi T, Umekawa S, eds., Japan Society for Composite Materials, North-Holland Publishing Company, 63: 279-286

Salpekar S A, Raju I S and O'Brien T K 1988 Strain energy release rate analysis of the end notched flexure specimen using the finite element method. J. Compos. Technol. Res. 10(4): 133-139

Sela N, Ishai O and Banks-Sills L 1989 The effect of adhesive thickness on inter-laminar fracture toughness of interleaved CFRP specimens. Int. J. Compos. 20(3): 257-264

Wang Y and Williams J G 1992 Corrections for mode II fracture toughness specimens of composite materials. Compos. Sci. Technol. 43: 251-256 
Whitney J M 1990 Analysis of inter-laminar mode II bending specimens using a higher order beam theory. J. Reinf. Plast. Compos. 9(6): 522-536

Whitney J M 1991 Elasticity analysis of orthotropic beams under concentrated loads. Compos. Sci. Technol. 22: $167-169$

Whitney J M and Browning C E 1998 On short beam shear tests for composite materials. Exp. Mech. 3: 290-294

Whitney J M and Pagano N J 1970 Shear deformation in heterogeneous anisotropic plates. J. Appl. Mech., Trans. ASME 37: 1031-1036

Whitney J M, Browning C E and Mair A 1974 Analysis of the flexure test for laminated composite materials. ASTM-STP-546 63: 252-259

Whitney J M, Gillespie Jr J W, Carlsson L A 1987 Singularity approach to the analysis of the end notch flexure specimen. In Proceedings of 2nd Conference on Composite Materials. Lancaster, PA: Technomic Publishing 63: 391-398 